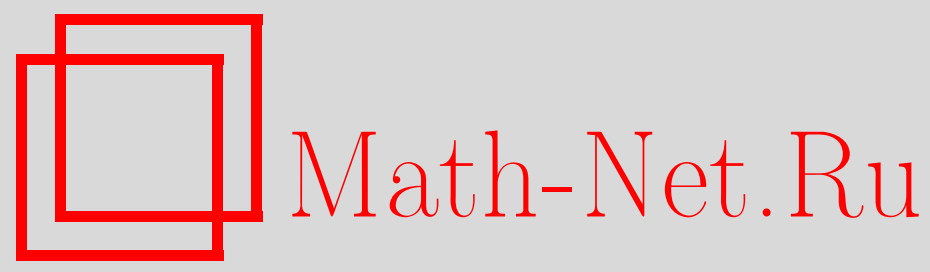

В. В. Белов, О. С. Доброхотов, С. Ю. Доброхотов, Изотропные торы, комплексный росток и индекс Маслова, нормальные формы и квазимоды многомерных спектральных задач, Матем. заметки, 2001, том 69, выпуск 4, 483-514

DOI: https://doi.org/10.4213/mzm519

Использование Общероссийского математического портала Math-Net.Ru подразумевает, что вы прочитали и согласны с пользовательским соглашением http://www . mathnet.ru/rus/agreement

Параметры загрузки:

IP : 18.234 .197 .8

26 апреля 2023 г., 13:01:26

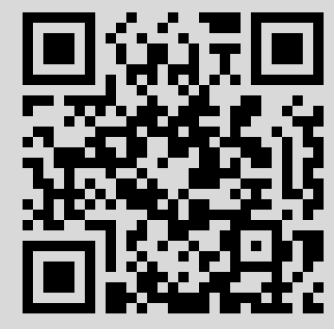




\section{ИЗОТРОПНЫЕ ТОРЫ, КОМПЛЕКСНЫЙ РОСТОК \\ И ИНДЕКС МАСЛОВА, НОРМАЛЬНЫЕ ФОРМЫ \\ И КВАЗИМОДЫ МНОГОМЕРНЫХ СПЕКТРАЛЬНЫХ ЗАДАЧ}

В.В. Белов, О.С. Доброхотов, С. Ю. Доброхотов

Более двадцати лет назад В.П. Маслов сформулировал вопрос о том, при каких условиях инвариантным изотропным неполномерным торам гамильтоновых систем могут быть сопоставлены последовательности асимптотических собственных значений и функций (спектральные серии) соответствуюших квантовомеханических и волновых операторов. В статье в терминах квадратичного приближения теории нормальных форм дан ответ на этот вопрос. Также обсуждаются вопросы об условиях квантования изотропных торов и их связь с топологическими, геометрическими и динамическими характеристиками (индексами Маслова, числами вращения, собственными числами динамических потоков и т.д.).

Библиографоия: 75 названий.

1. Формулировка задачи. Пусть гладкая функция Гамильтона $H(p, x)$ - символ [1]-[5] в существенном самосопряженного в $L^{2}\left(\mathbb{R}^{n}\right)$ оператора

$$
\widehat{H}=H\left(-i h \frac{\partial}{\partial x}, x\right) \equiv H\left(-i h \frac{\partial}{\partial x}, \frac{1}{2}\left(\frac{1}{x}+\stackrel{3}{x}\right)\right),
$$

где $h \ll 1$ - мальй параметр и значки $1,2,3$ над операторами $-i h \partial / \partial x, x$ означают порядок их действия (см. [1]-[5]) (т.е. операторы $-i h \partial / \partial x, x$ упорядочены по Вейлю). Предположим, что отвечающая $H(p, x)$ система Гамильтона интегрируема по Лиувиллю и допускает гладкое семейство инвариантных лагранжевых (лиувиллевых) торов $\Lambda^{n}(I)$, где $I=\left(I_{1}, \ldots, I_{n}\right) \subset \mathscr{M} \subset \mathbb{R}_{I}^{n}$ - переменные действия, отвечающие базисньм циклам $\Gamma_{j}, j=1, \ldots, n$, на $\Lambda^{n}(I) ; \mathscr{M}$ - некоторая открытая область. Тогда условие квантования Бора-Зоммерфельда-Маслова этого семейства

$$
I_{j}^{\nu}=\left(\nu_{j}+\frac{M_{j}}{4}\right) h+O\left(h^{2}\right), \quad\left(I_{1}^{\nu}, \ldots, I_{n}^{\nu}\right) \subset \mathscr{M},
$$

где $M_{j}$ - индекс Маслова цикла $\Gamma_{j}$ (см. [1], [3]) определяет асимптотику при $h \rightarrow 0$ некоторой подпоследовательности собственных значений спектральной задачи $\widehat{H} \psi=E \psi$, $\psi \in L_{2}\left(\mathbb{R}_{x}^{n}\right)$, по следующим формулам:

$$
E_{\nu}(h)=H\left(I^{\nu}(h)\right)+O\left(h^{2}\right), \quad H(I)=\left.H\right|_{\Lambda^{n}(I)} .
$$

Работа выполнена при частичной поддержке немецко-российского проекта DFG-PAH, грант № 436 RUS $113 / 572 / 1$. 
Отвечающие $E_{\nu}(h)$ асимптотические собственные функции $\psi_{\nu}(x, h)$ определяются с помощью (вещественного) канонического оператора Маслова: $\psi_{\nu}=\mathscr{K}_{\Lambda^{n}\left(I^{\nu}\right) \cdot 1}$ (см. [1], [3]); $E_{\nu}$ и $\psi_{\nu}$ удовлетворяют соотношению

$$
\widehat{H} \psi_{\nu}=E_{\nu} \psi_{\nu}+O\left(h^{\alpha}\right), \quad\left\|\psi_{\nu}\right\|=1 .
$$

Здесь $\alpha=2$ и $O\left(h^{\alpha}\right)$ - оценки невязки в норме $L_{2}\left(\mathbb{R}_{x}^{n}\right)$. При этом $\psi_{\nu}$ и $E_{\nu}$ локализованны в окрестности тора $\Lambda^{n}$ в том смысле, что $(k=n)$ :

$$
\lim _{h \rightarrow 0} \operatorname{supp} \psi_{\nu}=\pi_{x} \Lambda^{k} \quad \text { и } \quad \lim _{h \rightarrow 0} E_{\nu}(h)=\left.H\right|_{\Lambda^{k}},
$$

где $\pi_{x}$ - естественная проекция фазового пространства $\mathbb{R}_{p, x}^{2 n}$ на конфигурационное $\mathbb{R}_{x}^{n}$. Число $E_{\nu}$ и функцию $\psi_{\nu}$, удовлетворяющие (1.3), часто назьвают [6] квазимодой, а подпоследовательность $\left(E_{\nu}, \psi_{\nu}\right), I^{\nu} \in \mathscr{M},-$ спектральной серией.

Существуют различные модификации описанных "полномерных" квазиклассических асимптотик, в том числе аппелирующие к КАМ-теории и позволяющие существенно ослабить условия вполне интегрируемости (см. [7], [8]). По существу формулы $(1.1),(1.2)$ носят локальньй характер: допустимая область изменения параметров может быть достаточно малой, более того, достаточно даже иметь только один индивидуальньй тор с условно периодическим движением на нем, чтобы построить отвечающую ему некоторую "маломошную” спектральную серию. Если же гамильтонова система расслаивает все фазовое пространство на лиувиллевы торы, то формулы вида (1.1) аппроксимируют “почти” весь спектр оператора $\widehat{H}$ [7], [9], [10].

Вне конструкции вешественного канонического оператора Маслова остаются квазимоды, соответствующие сингулярньм многообразиям, представляющим собой “границы" между областями в фазовом пространстве (с одинаковыми топологическими и геометрическими характеристиками у соответствующих лиувиллевых торов, см., например, $[11],[12])$, и регулярным “неполномерным" многообразиям, изотропным торам $\Lambda^{k}$ размерности $k<n$ (вырожденным по отношению к лиувиллевым торам). $\mathrm{K}$ их числу относятся, в частности, точки покоя $(k=0)$ и замкнутые траектории $(k=1)$. Спектральные серии, отвечающие таким маломерным торам, могут быть получены уже с помощью комплексного канонического оператора Маслова - теории комплексного ростка [13]-[16]. Заметим, что существование неполномерных (или маломерных) торов и даже их $k$-мерных семейств $\Lambda^{k}(I), I=\left(I_{1}, \ldots, I_{k}\right) \subset \mathscr{M} \subset \mathbb{R}_{I}^{k}$ (с условно периодическим движением на них), не предполагает интегрируемости исходной гамильтоновой системы, но сопоставить им спектральные серии $\psi_{\nu}(x, h), E_{\nu}(h)$ или иначе - проквантовать $\Lambda^{k}$ в квазиклассическом приближении (в рамках комплексного канонического оператора Маслова) можно лишь при некоторых дополнительных условиях на торы $\Lambda^{k}$.

Этот факт содержится уже в пионерской работе Бабича [17], [18], посвященной квазимодам оператора Бельтрами-Лапласа, локализованным в окрестности замкнутой геодезической, и в работах [13], [19]-[21], касающихся замкнутых траекторий общих гамильтонианов. Один из основных вьводов, существенно опирающийся на теорию Флоке, состоит в следующем: необходимым и достаточным условием сопоставления замкнутым траекториям спектральных серий с помощью комплексного канонического оператора Маслова является свойство их орбитальной устойчивости в линейном приближении ${ }^{1}$. С геометрической точки зрения [13] это свойство означает, что может быть

\footnotetext{
${ }^{1} \mathrm{~B}$ работе [17] это свойство приводится как достаточное для замкнутой геодезической, необходимость его выполнения, включая случаи общих гамильтонианов, доказана в [13], [22] .
} 
построено векторное расслоение $\left[\Lambda^{k}, r^{n}\right]$ с базой $\Lambda^{k}(k=1)$ и слоями $r^{n}-$ некоторыми $n$-мерньпи плоскостями в комплексном фазовом пространстве; причем эти слои обладают подходящим свойством инвариантности, задаваемым системой в вариациях, которая порождается кривой $\Lambda^{1}$ и исходной гамильтоновой системой. Существование таких плоскостей, называемых комплексным ростком Маслова, является необходимым условием для построения квазимод соответствующих и $k$-мерным торам, $1<k<n$. Ситуация здесь, однако, является существенно отличной и более сложной по сравнению с ситуацией, отвечающей замкнутьм траекториям. Дело в том, что коэффищиенты системы в вариациях оказьваются, вообще говоря, почти периодическими функциями, и теория $\Phi$ локе здесь не работает. Поэтому сформулированные еще Масловым в книге [13] вопросы:

1) о необходимых и достаточных условия $x$ построения инвариантного комплексного ростка;

2) достаточно ли его существования для построения квазимод,

оказьваются абсолютно нетривиальными и до сих пор не имеют исчерпьвающего ответа. Ряд достаточных (и естественных) условий для ответов на оба вопроса были даны, в частности, в [13], [15], [16], [22]-[33]. Вообще, ответы на эти вопросы можно разделить на три категории:

(а) ответы, сформулированные на языке “квазиклассических геометрических асимптотик комплексного ростка" [13]-[16], [19], [20], т.е. на “геометрическом языке”;

(b) ответы на “языке связностей" [25], [27], [29]-[32];

(c) ответы на языке нормальных форм и динамических систем.

Формулировка этих условий в последней форме является на наш взгляд наиболее доступной для понимания и естественной для широкого круга специалистов, поскольку в ней используются лишь достаточно простые, общеизвестные, “устоявшиеся" и более привычные объекты из анализа и теории динамических систем (в отличие от объектов, используемых язьками ${ }^{2}(\mathrm{a})$ и (b)). Немаловажным обстоятельством является также тот факт, что теория нормальных форм в окрестности маломерных торов, а также примькающая к ней теория обыкновенных уравнений с условно периодическими коэффициентами была существенно продвинута за последние годы. Среди огромного количества публикаций упомянем монографии [34]-[36] и “этапные" недавно вьшшедшие статьи [37]-[47], в которых можно найти соответствующую практически полную библиографию.

Цель этой работы - дать ответы на сформулированные вьше вопросы на язьке квадратичного приближения теории нормальных форм и теории динамических систем при следующем дополнительном условии на торы $\Lambda^{k}$.

УСЛовИЕ і). Нормальное симплектическое расслоение $N\left(\Lambda^{k}\right)$ над торами $\Lambda_{\text {яв- }}^{k}$ ляется тривиальным.

Заметим, что до настоящего времени такой язык в теории комплексного ростка практически не использовался. Мы даем полный ответ (необходимыеи достаточные условия) на сформулированные вопросы 1), 2) в том смысле, что их решение переносится в

\footnotetext{
${ }^{2}$ Разумеется, соединение всех упомянутых результатов существенно помогает в решении рассматриваемой задачи.
} 
плоскость теории нормальных форм, динамических систем и уравнений с условно периодическими коэффищиентами (это приводит к нескольким, повидимому, новым постановкам задач). Попутно мы проясним вопрос об аналогах формул (1.1), (1.2) в неполномерном случае и о соответствуюших топологических характеристиках. Сначала в пш. 2-7 мы формулируем основные результаты, а технические детали доказательств, приводимых ниже утверждений, мы вьносим в отдельный раздел - п. 8. При этом мы приводим формулы лишь для асимптотических собственных значений, опуская формулы для асимптотических собственных функций, которые можно найти, в частности, в [13]-[16].

2. Инвариантные изотропные торы с комплексным ростком. Структура решений системы в вариациях. Пусть в $2 n$-мерном фазовом пространстве (для простоты евклидовом) $\mathbb{R}_{p, x}^{2 n}$ со стандартной симплектической 2 -формой $\omega^{2}=d p \wedge d x$ задана функция Гамильтона

$$
H=H(y) \equiv H(p, x) \in C^{\infty}\left(\mathbb{R}_{p, x}^{2 n}\right), \quad y=(p, x),
$$

такая, что соответствующая гамильтонова система

$$
\dot{p}=-H_{x}, \quad \dot{x}=H_{p} \Longleftrightarrow \dot{y}=J H_{y}
$$

допускает (по крайней мере один) инвариантньй $k$-мерньй изотропньй тор

$$
\begin{aligned}
\Lambda^{k}= & \left\{(p, x) \in \mathbb{R}^{2 n}, p=P(\varphi), x=X(\varphi)\right. \\
& \left.\varphi=\left(\varphi_{1}, \varphi_{2}, \ldots, \varphi_{k}\right), \varphi_{j} \in[0,2 \pi], j=1, \ldots, k\right\}
\end{aligned}
$$

с условно периодическим движением на нем. Здесь гладкие вектор-функции $P(\varphi), X(\varphi)$ - $2 \pi$-периодические по каждому аргументу;

$$
J=\left(\begin{array}{cc}
0 & -E_{n} \\
E_{n} & 0
\end{array}\right)
$$

$E_{n}$ - единичная $(n \times n)$-матрица. Через $O$ мы обозначаем матрицы с нулевыми элементами подходящего размера, и под $p, P, x, X, y, Y$ мы понимаем вектор-столбцы соответствующей размерности.

Условие изотропности означает, что в каждой точке $\varphi \in \Lambda^{k}\left((P(\varphi), X(\varphi)) \in \Lambda^{k}\right)$ все линейно независимые касательные векторы

$$
Y_{\varphi_{j}}=\left(\begin{array}{l}
\partial P / \partial \varphi_{j} \\
\partial X / \partial \varphi_{j}
\end{array}\right)
$$

косоортогональны друг другу:

$$
\omega^{2}\left(Y_{\varphi_{j}}, Y_{\varphi_{i}}\right)=\left\{Y_{\varphi_{j}}, Y_{\varphi_{i}}\right\}=\left\langle Y_{\varphi_{j}}, J Y_{\varphi_{i}}\right\rangle_{\mathbb{R}^{2 n}}=0, \quad i, j=1,2, \ldots, k
$$

Здесь и далее $\langle\cdot, \cdot\rangle$ - евклидово скалярное произведение в соответствующей размерности. Напомним [3], [48], что размерность $k$ не превосходит $n$; при $k=n$ изотропные торы назьваются лагранжсевы.ми. В дальнейшем мы всегда будем считать, что $k<n$. 
Инвариантность тора $\Lambda^{k}$ с условно периодическим движением означает, что траектории гамильтоновой системы (2.1) $\gamma_{\varphi}(t), \varphi \in \Lambda^{k}, t \in \mathbb{R}$, на нем имеют вид

$$
\gamma_{\varphi}(t)=\{y, y=Y(\omega t+\varphi)=(P(\omega t+\varphi), X(\omega t+\varphi))\}
$$

где $\omega=\left(\omega_{1}, \ldots, \omega_{k}\right) \in \mathbb{R}^{k}-$ вектор "частот".

Гамильтониан $H(y)=H(p, x)$ и торы $\Lambda^{k}$ порождают линеаризованную гамильтонову систему - систему в вариациях в окрестности $\Lambda^{k}$. В комплексификации ${ }^{\mathbb{C}} T \mathbb{R}_{p, x}^{2 n}$ касательного пространства к $\mathbb{R}_{p, x}^{2 n}$ в координатах $v={ }^{t}(w, z)\left(\right.$ координаты $w={ }^{t}\left(w_{1}, \ldots, w_{n}\right)$ $\in \mathbb{C}^{n}$ соответствуют импульсам $p$ и $z={ }^{t}\left(z_{1}, \ldots, z_{n}\right) \in \mathbb{C}^{n}-$ координатам $\left.x\right)$ на ${ }^{\mathbb{C}} T \mathbb{R}_{p, x}^{2 n}$ $\cong \mathbb{C}_{w, z}^{2 n}$ для каждой траектории $\gamma_{\varphi}(t) \in \Lambda^{k}$ эта система имеет вид

$$
\left(\begin{array}{c}
\dot{w} \\
\dot{z}
\end{array}\right)=\left.\left(\begin{array}{cc}
H_{p x} & H_{p p} \\
-H_{x x} & -H_{x p}
\end{array}\right)\right|_{\gamma_{\varphi(t)}}\left(\begin{array}{c}
w \\
z
\end{array}\right) \Longleftrightarrow \dot{v}=J H_{y y}(Y(\omega t+\varphi)) v
$$

где

$$
\left(\begin{array}{c}
w(t, \varphi) \\
z(t, \varphi)
\end{array}\right) \in{ }^{\mathbb{C}} T_{\gamma(t)} \mathbb{R}_{p, x}^{2 n}
$$

$H_{x x}, H_{p p}, H_{p x}={ }^{t} H_{x p}-(n \times n)$-матрицы из вторых производных функции $H$, например,

$$
H_{x p}=\left(\frac{\partial^{2} H}{\partial x_{i} \partial p_{j}}\right) \text {. }
$$

Индекс $t$ слева вверху здесь и далее означает транспонирование.

Напомним [13], что комплексным ростком $r^{n}$ надизотропным тором $\Lambda^{k}$ назьвается семейство комплексных плоскостей $r^{n}(\varphi) \in \mathbb{C}_{w, z}^{2 n}$, гладко зависящих от точки $\varphi \in \Lambda^{k}$, удовлетворяющих условиям:

(1) $r^{n}(\varphi)$ содержит комплексификацию касательного пространства в точке $\varphi \in \Lambda^{k}$, т.е. ${ }^{\mathbb{C}} T_{\varphi} \Lambda^{k} \subset r^{n}(\varphi)$;

(2) $r^{n}(\varphi)$ - лагранжева плоскость, т.е. $\left\{\xi^{\prime}, \xi^{\prime \prime}\right\}=0$ для всех $\xi^{\prime}, \xi^{\prime \prime} \in r^{n}(\varphi)$;

(3) $r^{n}(\varphi)$ - диссипативная плоскость, т.е. $\frac{1}{2 i}\{\xi, \bar{\xi}\} \geqslant 0$ и $\{\xi, \bar{\xi}\}=0 \Longleftrightarrow \xi \in{ }^{\mathbb{C}} T_{\varphi} \Lambda^{k}$.

Здесь и ниже через $\left\{\xi^{\prime}, \xi^{\prime \prime}\right\}$ обозначено кососкалярное произведение $2 n$-мерных комплексных векторов: $\left\{\xi^{\prime}, \xi^{\prime \prime}\right\}=\left\langle\xi^{\prime}, J \xi^{\prime \prime}\right\rangle_{\mathbb{R}^{2 n}}$, через $\bar{\xi}$ - вектор, комплексно-сопряженньй вектору $\xi$. Изотропньй тор (база) $\Lambda^{k}$ и плоскости $r^{n}(\varphi)$ (слои) образуют векторное расслоение $\left[\Lambda^{k}, r^{n}\right]$.

Комплексньй росток назьвается инвариантным, если

$$
d g_{H}^{t} r^{n}(\varphi)=r^{n}\left(g_{H}^{t}(\varphi)\right), \quad \varphi \in \Lambda^{k},
$$

где $g_{H}^{t}$ - фазовьй поток в $\mathbb{R}_{p, x}^{2 n}$, отвечающий системе Гамильтона $(2.1)$, a $d g_{H}^{t}$ - однопараметрическое семейство симплектических преобразований в $\mathbb{C}_{T} \mathbb{R}_{p, x}^{2 n}$, порожденных сдвигами вдоль траекторий линейной гамильтоновой системы (2.2). Из условия і) на изотропньй тор $\Lambda^{k}$ следует, что глобально слои $r^{n}(\varphi), \varphi \in \Lambda^{k}$, представляются в виде $r^{n}={ }^{\mathbb{C}} T \Lambda^{k} \oplus \tilde{r}^{n-k}$, где $(n-k)$-комплексномерные плоскости $\tilde{r}^{n-k}(\varphi)$ удовлетворяют условиям:

$1^{\prime} .\left\{\xi^{0}, \xi^{\prime}\right\}=0 \forall \xi^{0} \in{ }^{\mathbb{C}} T_{\varphi} \Lambda^{k}, \xi^{\prime} \in \tilde{r}^{n-k}(\varphi) ;$

$2^{\prime} .\left\{\xi^{\prime}, \xi^{\prime \prime}\right\}=0 \forall \xi^{\prime}, \xi^{\prime \prime} \in \tilde{r}^{n-k}(\varphi)$;

$3^{\prime} \cdot \frac{1}{2 i}\{\xi, \bar{\xi}\}>0 \forall \xi \in \tilde{r}^{n-k}(\varphi)$. 
Плоскости $\tilde{r}^{n-k}(\varphi)$ будем называть (допуская некоторую некорректность в терминологии) фактором комплексного ростка в точке $\varphi$ по (комплексифицированному) касательному пространству к $\Lambda^{k}$ в этой точке ${ }^{3}$.

В силу инвариантности $\Lambda^{k}$ и $r^{n}$ фактор комплексного ростка $\tilde{r}^{n-k}=r^{n} / \mathbb{C}_{T} \Lambda^{k}$ является также инвариантньм, т.е. выполнено условие

$$
4^{\prime} . d g_{H}^{t}\left(\tilde{r}^{n-k}(\varphi)\right)=\tilde{r}^{n-k}\left(g_{H}^{t}(\varphi)\right), \varphi \in \Lambda^{k} .
$$

Здесь $d g_{H}^{t}$ - сужение действия линейной гамильтоновой системы (2.2) на расслоение $E\left(\Lambda^{k}\right)$, косоортогональное к ${ }^{\mathbb{C}} T \Lambda^{k} ; E\left(\Lambda^{k}\right)=N\left(\Lambda^{k}\right) \oplus{ }^{\mathbb{C}} T \Lambda^{k}$, которое в силу условия і) является также тривиальным.

В предположениии і) условия существования инвариантного комплексного ростка $r^{n}$ (фактора $\tilde{r}^{n-k}$ ) формулируются в виде необходимых и достаточных условийна структуру матрищы Коши $\Phi(t, \varphi), t \in \mathbb{R}, \varphi \in \Lambda^{k}$, системы в вариациях (2.2). Во-первых, заметим, что из тривиальности расслоений $N\left(\Lambda^{k}\right)$ и $E\left(\Lambda^{k}\right)$ следует, что матрища $\Phi(t, \varphi)$ имеет вид

$$
\Phi(t, \varphi)=\left(Z(t, \varphi), \bar{Z}(t, \varphi), Y_{\varphi}(\varphi+\omega t), N(t, \varphi)\right) .
$$

Здесь $Z(t, \varphi), \bar{Z}(t, \varphi)$ - гладкие $((2 n) \times(n-k))$-комплексные матрищы; $((2 n) \times k)$-вешественная матрища $Y_{\varphi}(\varphi)$ - матрица на торе (т.е. она $2 \pi$-периодична по каждому $\varphi_{1}, \ldots, \varphi_{k}$ ), составленная из вектор-столбцов $Y_{\varphi_{j}}(\varphi)$ касательных векторов к $\Lambda^{k}$ в точке $\varphi ;$ и $N(t, \varphi)$ - $((2 n) \times k)$-вещественная матрица. При этом указанные матрищы удовлетворяют соотношениям ${ }^{4}$ :

$$
{ }^{t} Z J Y_{\varphi}={ }^{t} \bar{Z} J Y_{\varphi}=0, \quad{ }^{t} Z J N={ }^{t} \bar{Z} J N=0, \quad{ }^{t} Y_{\varphi} J N=E_{k}
$$

Требование существования комплексного ростка уточняет структуру матрицы $\Phi(t, \varphi)$ вида (2.3).

Лемма 1. Пусть выполнено условие і). Тогда инвариантный комплексный росток $r^{n}$ на изотропном инвариантном торе $\Lambda^{k}$ существует тогда и только тогда, когда матрица Кочи $\Phi(t, \varphi)$ системы в вариациях (2.2) может быть представлена в виде:

$$
\Phi(t, \varphi)=\left(V(\varphi+\omega t) U(t, \varphi), \bar{V}(\varphi+\omega t) \bar{U}(t, \varphi), Y_{\varphi}(\varphi+\omega t), N(t, \varphi)\right) .
$$

Здесь $((2 n) \times k)$-вещественные матрицы $Y_{\varphi}$ u $N$ mе же, что и в $(2.4) ; V(\varphi)-$ $((2 n) \times(n-k))$-комплексная матрица на торе $\Lambda^{k} ; U(t, \varphi)-((n-k) \times(n-k))$-унитарная матрича, $U(0, \varphi)=E_{n-k}$ (которая определена ниже в следствии из теоремы 1). Все они гладко зависят от $\varphi \in \Lambda^{k} u t \in \mathbb{R}$ и обладают свойствами (ср. $c(2.4))$ :

$$
\begin{gathered}
{ }^{t} V J Y_{\varphi}={ }^{t} \bar{V} J Y_{\varphi}=0, \quad{ }^{t} V J Y={ }^{t} \bar{V} J N=0, \\
{ }^{t} \bar{V} J V=2 i E_{n-k}, \quad{ }^{t} V J V=0, \quad{ }^{t} Y_{\varphi} J N=E_{k} .
\end{gathered}
$$

Вектор-столбцы матрицы $\left(V(\varphi), Y_{\varphi}(\varphi)\right)$ задают один из возмохнны базисов на комплексном ростке: плоскость $r^{n}(\varphi)$ может быть задана в каждой точке $\varphi \in \Lambda^{k}$ формулой

$$
r^{n}(\varphi)=\left\{{ }^{t}(w, z) \in \mathbb{C}_{w, z}^{2 n},\left(\begin{array}{c}
w \\
z
\end{array}\right)=\left(V(\varphi), Y_{\varphi}(\varphi)\right) \alpha, \alpha \in \mathbb{C}^{n}\right\}
$$

\footnotetext{
${ }^{3} \mathrm{~B}[14] \tilde{r}^{n-k}$ называется минимальным ростком.

${ }^{4}$ Матрица $N(t, \varphi)$ может быть явным образом выражена через матрицы $Z, \bar{Z}$ и $Y_{\varphi}$ (см. [49]).
} 


\section{3. Нормальная форма гамильтониана в окрестности инвариантного $k$-ме-} рного изотропного тора $\Lambda^{k}$ с комплексным ростком. Рассмотрим вначале случай, когда тор $\Lambda^{k}$ принадлежит гладкому $k$-параметрическому семейству инвариантньх торов $\Lambda^{k}(I)$ при $I=I_{0}$, где $I=\left(I_{1}, I_{2}, \ldots, I_{k}\right)$ - переменные действия, сопряженные угловым переменньм $\varphi=\left(\varphi_{1}, \varphi_{2}, \ldots, \varphi_{k}\right)$, которые задают тор

$$
\Lambda^{k}(I)=\{(p, x), p=P(\varphi, I), x=X(\varphi, I)\},
$$

$I_{j}=(2 \pi)^{-1} \oint_{\Gamma_{j}} p d x$, где $\Gamma_{j}=\Gamma_{j}(I)-j$-й цикл по переменной $\varphi_{j}$ на $\Lambda^{k}(I)$, т.e. $\varphi_{i}=$ const, $i \neq j, j, i=1,2, \ldots, k$. Гладкость семейства $\Lambda^{k}(I)$ означает, что $2 \pi$-периодические по каждому аргументу $\varphi_{j} \in[0,2 \pi], j=1,2, \ldots, k$, вектор-функции $P(\varphi, I), X(\varphi, I)$ и вектор частот $\omega(I)=\left(\omega_{1}(I), \ldots, \omega_{k}(I)\right)$ условно периодического движения на торе $\Lambda^{k}(I)$ гладко зависят от $I \subset M_{I}$, где $M_{I}$ - некоторая область в $\mathbb{R}_{I}^{k}$. Комплексньй росток $r^{n}(I)$ над изотропным тором $\Lambda^{k}(I)$ может не существовать для каждого $I \in M_{I}$. Условие (2.5) может вырезать из области $M_{I}$ достаточно сложное подмножество, которое обозначим $M_{I}^{r}$. Чтобы упростить ситуацию, зафиксируем точку $I_{0}$ и соответствующий тор $\Lambda^{k}\left(I_{0}\right)$ и предположим, что комплексньй росток также существует в некоторой окрестности $I_{0}$. Для упрощения обозначений обозначим эту окрестность через $M_{I}$ (тогда $\left.M_{I}^{r}=M_{I}\right)$.

Семейство торов $\Lambda^{k}(I)$ с комплексным ростком $r^{n}(I)$ над $\Lambda^{k}(I), I \in M_{I}$, порождает в окрестности тора $\Lambda^{k}\left(I_{0}\right)$ “почти” симплектические координаты $y^{\prime}=(I, \varphi, \xi, \bar{\xi})$, где $\xi, \bar{\xi} \in \mathbb{C}^{n-k}$ ( $\xi$ и $\bar{\xi}$-комплексные “нормальные" координаты). Обозначим через $y$ евклидовы координаты фазового пространства $\mathbb{R}_{p, x}^{2 n}, y={ }^{t}(p, x)$; тогда переход от координат $y^{\prime}$ к координатам $y$ осуществляется по следующим формулам:

$$
y=Y(\varphi, I)+\frac{1}{2}(V(\varphi, I) \xi+\bar{V}(\varphi, I) \bar{\xi})+Y_{I}(\varphi, I) g(\varphi, I, \xi, \bar{\xi}) .
$$

Здесь вектор-функция $Y(\varphi, I)={ }^{t}(P(\varphi, I), X(\varphi, I))$ задает тор $\Lambda^{k}(I)$, комплексная $((2 n) \times(n-k))$-матрица $V(\varphi, I)$ определяет базис на факторе комплексного ростка $\tilde{r}^{n-k}(\varphi, I), \varphi \in \Lambda^{k}(I), Y_{I}(\varphi, I)-((2 n) \times k)$-матрица $\partial Y(\varphi, I) / \partial I$ и $g-k$-мерная вектор-функция с компонентами (ср. с [34, гл. I, II])

$$
g_{j}(\varphi, I, \xi, \bar{\xi})=-\frac{1}{4}\left\langle\left(\begin{array}{c}
\xi \\
\bar{\xi}
\end{array}\right),\left(\begin{array}{ll}
{ }^{t} V J V_{\varphi_{j}} & { }^{t} V J \bar{V}_{\varphi_{j}} \\
{ }^{t} \bar{V} J V_{\varphi_{j}} & { }^{t} \bar{V} J \bar{V}_{\varphi_{j}}
\end{array}\right)\left(\begin{array}{c}
\xi \\
\bar{\xi}
\end{array}\right)\right\rangle, \quad j=1,2, \ldots, k,
$$

где

$$
V_{\varphi_{j}}=V_{\varphi_{j}}(\varphi, I)=\frac{\partial}{\partial \varphi_{j}} V\left(\varphi_{1}, \ldots, \varphi_{j}, \ldots, \varphi_{k}, I\right) .
$$

Теорема 1. Пусть выполнено условие і). Инвариантный комплексный росток $r^{n}$ на изотропном инвариантном торе $\Lambda^{k}$ существует тогда и только тогда, когда найдется комплексная $((2 n) \times(n-k))$-матрица $V(\varphi, I)$ со свойствами (2.6) такая, что

1) в окрестности тора $\Lambda^{k}\left(I_{0}\right)$ замена переменных $(3.1),(3.2)$ является "почти канонической", т.е.

$$
d p \wedge d x=d I \wedge d \varphi+2 i d \xi \wedge d \bar{\xi}+O\left(\|\xi\|^{3}\right)
$$


2) в координатах $y^{\prime}=(\varphi, I, \xi, \bar{\xi})$ в малой окрестности тора $\Lambda^{k}\left(I_{0}\right)($ при $\|\xi\|=$ $\sqrt{\langle\xi, \bar{\xi}\rangle}<\varepsilon, \varepsilon$ достаточно мало) гамильтониан $H\left(y^{\prime}\right)$ имеет вид (нормальную форму)

$$
H\left(y^{\prime}\right)=\left.H\right|_{\Lambda^{k}(I)}+\frac{1}{2}\langle\bar{\xi}, A(\varphi, I) \xi\rangle+O\left(\|\xi\|^{3}\right)
$$

где заданная на торах $\Lambda^{k}(I)$ эрмитова $((n-k) \times(n-k))$-матрица $A(\varphi, I)$ определяется формулой

$$
A(\varphi, I)=\frac{1}{2}\left({ }^{t} \bar{V}(\varphi, I) J\left\langle\omega(I), \frac{\partial}{\partial \varphi}\right\rangle V(\varphi, I)+{ }^{t} \bar{V} H_{y y}(Y(\varphi, I)) V\right) .
$$

Здесь $H_{y y}-((2 n) \times(2 n))$-матрица вторых производных гамильтониана $H(y)(y=$ $\left.{ }^{t}(p, x)\right), \omega(I)-k$-мерный вектор “частот" условно-периодического движения на mope $\Lambda^{k}(I)$.

СлЕДСТВИЕ. В координатах $y^{\prime}$ линеаризованная в окрестности тора $\Lambda^{k}(I)$ гамильтонова система (2.2) (система в вариациях) относительно переменных $\xi$ принимает вид

$$
\dot{\xi}=i A(\omega(I) t+\varphi, I) \xi, \quad \xi(\varphi, t) \in \mathbb{C}^{n-k} .
$$

ЗАмечАниЕ 1. Содержательные факты в теореме состоят в том, что, во-первых, в квадратичной части разложения гамильтониана $H$ по "нормальньм" переменньм $\xi$ и $\bar{\xi}$ отсутствуют члены вида $\xi_{i} \xi_{j}$ и $\bar{\xi}_{i} \bar{\xi}_{j}, i, j=1, \ldots, n-k$, и, во-вторых, что матрища $A(\varphi, I)$ эрмитова. Из последнего факта, в частности, следует, что система в вариациях (3.6) сохраняет положительно определенную эрмитову форму $\langle\xi, \bar{\xi}\rangle, \xi \in \mathbb{C}^{n-k}$, и, следовательно, ее матрища Коши является унитарной матрицей $U(t, \varphi)$, входящей в представление матрицы Коши (2.5) для полной системы в вариациях (2.2). Это означает, что тор $\Lambda^{k}(I)$ орбитально устойчив в линейном приближении. Нам не удалось найти в литературе ответ на следующий естественньй вопрос: следует ли из орбитальной устойчивости тора $\Lambda^{k}(I)$ представление гамильтониана в виде (3.4)?

ЗАмЕчАниЕ 2. Указанньй в п. 3) утверждения теоремы базис комплексного ростка, во-первых, неединственньй и, во-вторых, не является инвариантным относительно преобразования $d g_{H}^{t}$, а именно, $d g_{H}^{t} V(\varphi, I)=V(\varphi+\omega t) U(\varphi, I, t)$, где унитарная матрица $U(\varphi, I, t)$ - матрица Коши системы (3.6).

Мы уже отмечали, что подмножество $M_{I}^{r}$ существования комплексного ростка может быть весьма сложным. Вероятно, что окрестность, где можно ввести описанные координаты $y^{\prime}$, существует в очень редких ситуациях. Для квантовомеханических приложений это не необходимо, более того, для конструкции серии квазимод достаточно существования лишь единственного тора с комплексным ростком. Мы вернемся к этому вопросу в п. 7. С другой стороны, вероятно возможно доказать, что в некоторой окрестности тора $\Lambda^{k}\left(I_{0}\right)$ существует “почти" каноническоепреобразование, которое является гладким в смысле Уитни и дает представление (3.4). В любом случае всегда можно определить функцию $y(\varphi, I, \xi, \bar{\xi})(3.1)$ для $I \in M_{I}^{r}$ и вычислить гамильтониан $H(y(\varphi, I, \xi, \bar{\xi}))$ в виде (3.4), а также каноническое преобразование комплексного ростка для $I \in M_{I}^{r}$ в силу системы (3.6). 


\section{4. Приводимость уравнения в вариациях и почти инвариантные лагран-} жевы торы в окрестности изотропных торов с комплексным ростком. Для дальнейшего полезно ввести понятие почти инвариантного изотропного тора относительно заданного гамильтониана $H: \mathbb{R}_{y}^{2 n} \rightarrow \mathbb{R}, y={ }^{t}(p, x)$. Пусть $\Lambda^{k}(\varepsilon)$ - изотропные торы, гладко зависящие от набора параметров $\varepsilon=\left(\varepsilon_{1}, \varepsilon_{2}, \ldots, \varepsilon_{m}\right) \in U_{\delta_{0}}$, где $U_{\delta_{0}}-$ малая окрестность нуля в пространстве параметров $\mathbb{R}_{\varepsilon}^{m}$, т.е. множество, заданное соотношениями

$$
|\varepsilon|=\sqrt{\sum_{k=1}^{m} \varepsilon_{k}^{2}} \leqslant \delta_{0} \ll 1 ;
$$

причем эти торы имеют вид

$$
\Lambda^{k}(\varepsilon)=\left\{y \mid y=Y\left(\varphi_{1}, \varphi_{2}, \ldots, \varphi_{n}, \varepsilon\right), \varphi_{j} \in[0,2 \pi], j=1,2, \ldots, n\right\} .
$$

Здесь вектор-функция $Y(\varphi, \varepsilon), \varphi=\left(\varphi_{1}, \varphi_{2}, \ldots, \varphi_{k}\right),-2 \pi$-периодическая по каждому своему аргументу и $\left\langle Y_{\varphi_{j}}(\varphi, \varepsilon), J Y_{\varphi_{l}}(\varphi, \varepsilon)\right\rangle=0, j, l=1,2, \ldots, k$.

ОПРЕДЕЛЕНИЕ. Изотропньй тор $\Lambda^{k}(\varepsilon)$ называется почти инвариантным относительно гамильтониана $H(y)$ по $\bmod O\left(\varepsilon^{M+\delta}\right)$ при $|\varepsilon| \rightarrow 0(M \in \mathbb{N}, \delta>0)$, если существует $k$-мерный вектор (частот) $\omega$, зависящий, вообще говоря, от $\varepsilon$, такой, что функция $Y_{\varepsilon}=Y(\varphi+\omega t, \varepsilon)$ удовлетворяет системе Гамильтона по $\bmod O\left(\varepsilon^{M}\right)$ :

$$
\dot{Y}_{\varepsilon}=J H_{y}\left(Y_{\varepsilon}\right)+O\left(|\varepsilon|^{M}\right), \quad H\left(Y_{\varepsilon}\right)=\left.H\right|_{\Lambda^{n}(0)}+O\left(|\varepsilon|^{M+\delta}\right) \quad \text { при } \varepsilon \rightarrow 0,
$$

причем все оценки равномерны по $\varphi \in([0,2 \pi])^{n}$ и $t \in \mathbb{R}$. В случае $k=n$ получаем почти инвариантный лагранжев тор.

Рассмотрим теперь систему обькновенных дифференциальных уравнений (3.6) с квазипериодическими коэффициентами. Эта система индуцирована системой в вариациях (2.2) на семействе инвариантных изотропных торов $\Lambda^{k}(I)$ с комплексньп ростком $r^{n}(I)$, введенном в п. 2 .

В этом пункте будем рассматривать регуляный случай, т.е. случай, когда система в вариациях (3.6) является приводимой, и соответствующий комплексньй росток будем назьвать регулярным комплексньм ростком. Напомним понятие приводимости (см., например, [35]-[43]).

ОПРЕДЕЛЕниЕ. Система линейных обыкновенных дифференциальных уравнений (3.6) с квазипериодическими коэффищиентами является приводимой, если существует линейное невырожденное преобразование $\xi=U_{0}(\omega(I) t+\varphi, I) \tilde{\xi}$, приводящее эту систему к системе с постоянными коэффициентами.

Здесь $U_{0}(\varphi, I)$ - гладкая матричная функция на торе $\Lambda^{k}(I)$.

Обозначим через $\Gamma(I)$ (постоянную) матрицу приведенной системы. Очевидно, что $\Gamma(I)=\left(i U_{0}^{*} A U_{0}+U_{0}^{*} \dot{U}_{0}\right)$. В силу унитарности оператора Коши для системы (3.6) матрица $U_{0}=U_{0}(\omega t+\varphi, I)$ унитарна, а $\Gamma(I)$ - косоэрмитова матрица. Обозначим через $i \beta_{j}(I), j=1,2, \ldots, n-k$, собственные значения матрицы $\Gamma(I)$, отвечающие собственным векторам $f_{j}(I), j=1,2, \ldots, n-k$, и через $T(I)$ унитарную $((n-k) \times(n-k))$-матрицу, составленную из векторов $f_{j}(I), T(I)=\left[f_{1}(I), \ldots, f_{n-k}(I)\right]$. Тогда в собственном базисе матрицы $\Gamma(I)$ фундаментальная матрица системы (3.6) принимает вид 
$\widetilde{U}_{0}(\omega(I) t+\varphi, I) e^{i \hat{\beta} t}$, где $\hat{\beta}=\hat{\beta}(I)-((n-k) \times(n-k))$-диагональная матрища, $\hat{\beta}=$ $\operatorname{diag}\left(\beta_{j}(I)\right), \widetilde{U}_{0}=T^{-1} U_{0} T$. Числа $\beta_{j}(I)$ назьваются числами вращения приводимой системы (3.6) с квазипериодическими коэффициентами (аналог показателей $\Phi$ локе [41]-[43]).

ЗАмЕчАниЕ 3 . Если $k \leqslant 1$, то в силу теории Флоке система (3.6), как и исходная система в вариациях (2.2), всегда приводима. В другом “предельном" случае $k=n-1$ (3.6) - это скалярное уравнение, и при выполнении диофантова условия на частоты условно-периодического движения (см., например, [7], [34], [35], [51], а также п. 6) уравнение (3.6), как и система (2.2), также оказьваются приводимьми. Заметим, что в цитированных работах [35], [40], [45]-[47] условие приводимости в промежуточных случаях $1<k<n-1$, как правило, предполагается вьполненным, т.е. предполагается, что в уравнении (3.6) $A$ - постоянная матрица, и речь в основном идет о вычислении нормальных форм более высоких порядков.

Перейдем на плоскости $\tilde{r}^{n-k}(\varphi), \varphi \in \Lambda^{k}(I)$, к новым координатам $\tilde{\xi}, \tilde{\xi}=T^{-1}(I) \xi$. В новых координатах матрица системы (3.6) $\widetilde{A}=T^{-1} A T$, очевидно, будет определяться формулой $(3.5)$ при замене $V(\varphi, I)$ на $\widetilde{V}(\varphi, I) T(I)$, и система $(3.6)$ в этих координатах приводится к системе с постоянной диагональной матрицей $\hat{\beta}$ с помошью унитарной матрицы $\widetilde{U}_{0}(\omega t+\varphi, I)$. Ниже считаем, что эта замена координат проведена, и знак тильда при этом опускаем.

Рассмотрим теперь в фазовом пространстве $\mathbb{R}_{y}^{2 n}$ семейство многообразий, зависящих от параметров $I$ и $\rho, \rho=\left(\rho_{1}, \ldots, \rho_{l}, \ldots, \rho_{n-k}\right), \rho_{l}>0$, и задаваемых соотношением

$$
\Lambda^{n}(I, \rho)=\left\{y \mid y=Y(\varphi, \eta, I, \rho)=Y(\varphi, I)+\operatorname{Re}\left(V(\varphi, I) e^{i \hat{\eta}} \sqrt{\frac{\rho}{2}}\right)+Y_{I}(\varphi, I) f\right\} .
$$

Здесь вектор-функция $Y(\varphi, I)$ и матрища $V(\varphi, I)$ те же, что и вьше (см. п. $2,(3.1)), \hat{\eta}-$ диагональная $((n-k) \times(n-k))$-матрица, $\hat{\eta}=\operatorname{diag}\left(\eta_{l}\right), \eta_{l} \in[0,2 \pi], l=1,2, \ldots, n-k$, и $\sqrt{\rho / 2}-(n-k)$-мерный вектор с координатами $\sqrt{\rho_{l} / 2}$, где $\rho_{l} / 2 \geqslant 0, l=1,2, \ldots, n-k$, и через $f$ обозначен $k$-мерный вектор $f=g\left(\varphi, I, e^{i \hat{\eta}} \sqrt{\rho / 2}, e^{-i \hat{\eta}} \sqrt{\rho / 2}\right)$, где вектор $g$ определен в (3.2). Из теоремы 1 вытекает (cp. с [34], [35], [37])

СлЕДСТВИЕ. Пусть справедливы условия теоремы 1 и система (3.6) является приводимой. Тогда многообразие $\Lambda^{n}(I, \rho)$ есть $n$-мерный лагранжев тор, почти инвариантный по $\bmod O\left(|\rho|^{3 / 2}\right),|\rho| \rightarrow 0$, относительно гамильтониана $H(y) ; n р и$ этом

$$
\left.H(y)\right|_{\Lambda^{n}(I, \rho)}=\left.H\right|_{\Lambda^{k}(I)}+\sum_{l=1}^{n-k} \beta_{l}(I) \rho_{l}+O\left(|\rho|^{3 / 2}\right),
$$

әде $\beta_{l}(I), l=1,2, \ldots, n-k,-$ числа вращения системы (3.6).

5. Топологические характеристики - индексы Маслова изотропных торов с комплексным ростком. Их связь с индексами Маслова $\mathbb{C}$-лагранжевых многообразий и почти инвариантных лагранжевых торов. Хорошо известно [1], [3], что в задачах квазиклассического квантования изотропных инвариантных многообразий максимальной размерности $n$ (лагранжевых многообразий) существенную роль играют индексы Маслова циклов (замкнутых путей, не гомотопных точке) на лагранжевых многообразиях. 
При квантовании изотропных торов в рамках общей теории комплексного ростка Маслова в [2], [51], [52] было введено понятие индекса комплексного ростка на так называемом $\mathbb{C}$-лагранжевом многообразии. Индексы рассматриваемых здесь геометрических объектов - изотропных торов с комплексным ростком - в принципе могут быть получены из общей теории комплексного ростка [2], [50]. Однако такая редукция опирается на достаточно сложные рассуждения, и поэтому перенесение понятия индекса комплексного ростка из общей теории на рассматриваемьй случай требует дополнительных рассуждений и не является оптимальным.

Цель этого пункта - дать, основываясь на соображениях и определениях [2], [51], по-возможности максимально простое определение индекса комплексного ростка в рассматриваемой ситуации и апелируюшее лиш к объектам, введенным в зтой работе. Ниже мы также покажем, что при вьполнении условий п. 1 и теоремы 1 индексы комплексного ростка и индексы Маслова базисных циклов на почти инвариантном лагранжевом торе $\Lambda^{n}(I, \rho)(4.1)$ совпадают.

5.1. Индекс комплексного ростка. Согласно теореме 1 (утверждение 3)) базис на комплексном ростке $r^{n}(\varphi), \varphi \in \Lambda^{k}(I)$, может быть составлен из вектор-столбцов матрицы

$$
\left(\begin{array}{l}
B \\
C
\end{array}\right)=\left(V(\varphi), Y_{\varphi}(\varphi)\right)=\left(\begin{array}{cc}
\tilde{B}(\varphi) & P_{\varphi} \\
\tilde{C}(\varphi) & X_{\varphi}(\varphi)
\end{array}\right),
$$

где $\tilde{B}(\varphi)$ и $\tilde{C}(\varphi)-(n \times(n-k))$-блоки матрицы $V(\varphi)$, т.е. $B=\left(\tilde{B}(\varphi), P_{\varphi}(\varphi)\right), C=(\tilde{C}(\varphi)$, $\left.X_{\varphi}(\varphi)\right)$.

Введем теперь на торе $\Lambda^{k}(I)$ матрицу $C(\varphi)-i \varepsilon B(\varphi)$, где $\varepsilon>0$. Из аксиом комплексного ростка (условий лагранжевости и диссипативности) следует (см. ниже лемму 4 , a также [51]), что матрица $C(\varphi)-i \varepsilon B(\varphi)$ невырождена и поэтому определен якобиан

$$
J(\varphi)=\operatorname{det} C(\varphi)-i \varepsilon B(\varphi) .
$$

Пусть $\Gamma_{j}(I), j=1,2, \ldots, n-k,-$ цикл на торе $\Lambda^{k}(I)$, задаваемьй равенством

$$
\Gamma_{j}: \varphi_{j} \in[0,2 \pi], \varphi_{1}, \ldots, \varphi_{j-1}, \varphi_{j+1}, \ldots, \varphi_{k}=\text { const }
$$

ОПРЕДЕЛЕНИЕ. Целое число (не зависящее от $\varepsilon>0$ )

$$
\sigma_{j}=\left.\frac{1}{\pi} \operatorname{Arg} J(\varphi)\right|_{\varphi_{j}=\varphi_{j}^{0}} ^{\varphi_{j}=\varphi_{j}^{0}+2 \pi}
$$

назьвается индексом комплексного ростка для иикла $\Gamma_{j}(I)$ на изотропном торе $\Lambda^{k}(I)$.

Это определение индекса, вообще говоря, зависит от выбора базиса на комплексном ростке. При переходе к новому базису матрицы $\tilde{B}(\varphi)$ и $\tilde{C}(\varphi)$ умножаются на гладкую унитарную матрицу $\tilde{U}(\varphi)$, заданную на торе, что может привести в определении $(5.2)$ к изменению индекса на целое четное число. Важньм обстоятельством является тот факт, что индекс комплексного ростка с каким-либо фиксированным базисом является инвариантным при изменении базиса с помощю канонического преобразования $d g_{H}^{t}$ в силу системы в вариациях. Действительно, в этом случае

$$
d g_{H}^{t} V(\varphi)=V(\varphi+\omega(I) t) U(\varphi, I, t),
$$


где $U(\varphi, I, t)$ - матрища Коши системы (3.6). Так как $U(\varphi, I, t)$ - невырожденная и непрерьвная матрица на торе $\Lambda^{k}$, то приращение нормированного на $\pi$ аргумента ее детерминанта $\operatorname{det} U(\varphi, I, t)$ по циклам $\Gamma_{j}$ на $\Lambda^{k}(I)$ есть также непрерывная целочисленная функция $t$, которая при $t=0$ равна нулю. Эти рассуждения и дают указанную инвариантность. Произвол же в выборе матрицы $\tilde{V}(\varphi)$ в задаче о построении спектральных серий сводится к перенумерации собственных значений и аналогичен неоднозначному выбору угловых переменных на лагранжевых торах в полномерном случае.

5.2. $\mathbb{C}$-лагранжево многообразие и его индекс. Покажем, что индекс коплексного ростка, определенный в 5.1, совпадает с индексом $\mathbb{C}$-лагранжевого многообразия [51], ассоциированного с изотропным тором $\Lambda^{k}(I)$ и комплексным ростком $r^{n}(I)$ на $\Lambda^{k}(I)$. Согласно [51] $\mathbb{C}$-лагранжево многообразие есть $n$-вещественномерное многообразие в комплексном фазовом пространстве $\mathbb{C}_{w, z}^{2 n}$ с координатами $w \in \mathbb{C}^{n}$ и $z \in \mathbb{C}^{n}$. В рассматриваемом случае оно определяется формулами

$$
\begin{gathered}
w=W(\varphi, I)=P(\varphi, I)+\tilde{\tilde{B}}(\varphi, I) \alpha+O\left(|\alpha|^{2}\right), \\
z=Z(\varphi, I)=X(\varphi, I)+\tilde{\tilde{C}}(\varphi, I) \alpha+O\left(|\alpha|^{2}\right),
\end{gathered}
$$

где вектор-столбцы $(n \times n)$-матрищ $\tilde{\tilde{B}}(\varphi, I), \tilde{\tilde{C}}(\varphi, I)$, заданных на торе, образуют некоторьй базис на комплексном ростке $r^{n}(\varphi)$ и $\alpha-$ вещественные координаты, т.е. $\alpha=$ $\left(\alpha_{1}, \ldots, \alpha_{n}\right) \in \mathbb{R}^{n}$. Конкретный вид членов $O\left(|\alpha|^{2}\right)$ обеспечивает вьполнение лагранжевости введенного $\mathbb{C}$-лагранжевого многообразия, но не влияет на последующие определения. Это многообразие порождает матрицу

$$
\frac{\partial Z}{\partial \alpha}(\varphi, I)-\left.i \varepsilon \frac{\partial W}{\partial \alpha}(\varphi, I)\right|_{\alpha=0},
$$

которая совпадает с введенной выше матрищей $C-i \varepsilon B$, если выбрать $\tilde{\tilde{B}}=B, \tilde{\tilde{C}}=C$. Нормированное на $\pi$ приращение аргумента детерминанта этой матрицы по циклам $\Gamma_{j}(I)$ называется (см. [51]) индексом Маслова цикла $\Gamma_{j}(I)$ на $\mathbb{C}$-лагранжевом многообразии. Его свойства подробно описаны в книгах [2], [51]. Ясно, что введенный выше в (5.2) индекс комплексного ростка цикла $\Gamma_{j}(I)$ совпадает с индексом цикла $\Gamma_{j}$ на $\mathbb{C}$-лагранжевом многообразии. Заметим, что определение $\mathbb{C}$-лагранжева многообразия зависит от выбора базиса (матрищ $\tilde{\tilde{B}}(\varphi, I), \tilde{\tilde{C}}(\varphi, I))$ на комплексном ростке и оно не является инвариантньм (в отличие от изотропного тора с комплексным ростком) относительно канонического преобразования $\left(g_{H}^{t}, d g_{H}^{t}\right)$, для этого требуется комплексность координат $\alpha$. Тем не менее все полученные таким образом $\mathbb{C}$-лагранжевы многообразия пересекаются по тору $\Lambda^{k}$, и можно сравнивать индексы одинаковых циклов, построенные по разным $\mathbb{C}$-лагранжевым многообразиям, полученных с помощью таких канонических преобразований. Из приведенных вьше рассуждений следует, что эти индексы совпадают.

5.3. Связь индексов (регулярного) комплексного ростка в приводимом случае с индексом Маслова почти инвариантных лагранжевых торов. Напомним определение индекса Маслова замкнутого пути на лагранжевом торе $\Lambda^{n}(I, \rho)(4.1)$, который в исходных канонических координатах $p$ и $x$ задается формулами

$$
\begin{aligned}
& p=\widetilde{P}(\varphi, \eta, I, \rho)=P(\varphi, I)+\operatorname{Re}\left(\widetilde{B}(\varphi, I) e^{i \hat{\eta}} \sqrt{\rho}\right)+P_{I}(\varphi, I) f, \\
& x=\widetilde{X}(\varphi, \eta, I, \rho)=X(\varphi, I)+\operatorname{Re}\left(\widetilde{C}(\varphi, I) e^{i \hat{\eta}} \sqrt{\rho}\right)+X_{I}(\varphi, I) f .
\end{aligned}
$$


Здесь вектор-функции $P(\varphi, I)$ и $X(\varphi, I)$ задают изотропный тор $\Lambda^{k}(I)$, a $(n \times(n-k))$-матрищы $\widetilde{B}(\varphi, I)$ и $\widetilde{C}(\varphi, I)$ - блоки $((2 n) \times(n-k))$-матрищы $V(\varphi, I)$, определяющей базис на факторе комплексного ростка $\tilde{r}^{n-k}(I)$. Построим матрицу ${ }^{5}$

$$
D(\varphi, \eta)=\frac{\partial \tilde{X}}{\partial(\varphi, \eta)}-i \varepsilon \frac{\partial \widetilde{P}}{\partial(\varphi, \eta)}
$$

где $\varepsilon$ - вещественный параметр, $\varepsilon>0$. В силу свойства лагранжевости $(n$-мерного) тора $\Lambda^{n}(I, \rho)$ эта матрица невырождена при достаточно малых $\rho$ (см. доказательство леммы 2) и, следовательно, можно определить аргумент якобиана

$$
J(\varphi, \eta)=\operatorname{det}\left(\frac{\partial \widetilde{X}}{\partial(\varphi, \eta)}-i \varepsilon \frac{\partial \widetilde{P}}{\partial(\varphi, \eta)}\right) \neq 0
$$

и его прирашение $\Delta(l, \varepsilon)=\Delta_{l} \operatorname{Arg} J(\varphi, \eta)$ вдоль пути $l$ на $\Lambda^{n}$. Если $l$ - замкнутьй путь, то ясно, что $\Delta(l, \varepsilon)$ не зависит от $\varepsilon>0$, а зависит только от $l$. Тогда индекс Маслова замкнутого пути $l$ на $\Lambda^{n}-\operatorname{Ind} l$ определяется формулой Ind $l=\Delta(l) / \pi$.

Обозначим через $\Gamma_{j}(\rho, I)=\Gamma_{j}, j=1,2, \ldots, k$, и $\gamma_{l}(\rho, I)=\gamma_{l}, l=1,2, \ldots, n-k, \varphi$ и $\eta$-циклы на торе $\Lambda^{n}(I, \rho)(4.1)$, задаваемые равенствами

$$
\Gamma_{j}: \varphi_{j} \in[0,2 \pi], \varphi_{1}, \ldots, \varphi_{j-1}, \varphi_{j+1}, \ldots, \varphi_{k}=\text { const, } \eta_{1}=\cdots=\eta_{n-k}=\text { const }
$$

и

$$
\gamma_{l}: \eta_{l} \in[0,2 \pi], \quad \varphi_{1}=\cdots=\varphi_{k}=\mathrm{const}, \eta_{1}, \ldots, \eta_{l-1}, \eta_{l+1}, \ldots, \eta_{n-k}=\mathrm{const}
$$

соответственно. При $\rho \rightarrow 0 \varphi$-циклы $\Gamma_{j}$ переходят в циклы $\Gamma_{j}(0, I)=\Gamma_{j}(I)$ на изотропном торе $\Lambda^{k}(I)$, а циклы $\gamma_{l}$ по “ростковым", т.е. "нормальньм" к $\Lambda^{k}(I)$, переменным стягиваются в соответствуюшие точки $\varphi$ на $\Lambda^{k}(I)$, при этом параметры $I$ и $\rho$ семейства $\Lambda^{n}(I, \rho)$ являются переменными действия, сопряженньпи с углами $\varphi$ и $\eta$ на торе $\Lambda^{n}(I, \rho)$ соответственно. Из формул $(24)$, задающих тор $\Lambda^{n}(I, \rho)$, следует, что

$$
\begin{gathered}
\oint_{\Gamma_{j}(\rho, I) \in \Lambda^{n}(I, \rho)} p d x=\oint_{\Gamma_{j}(I) \in \Lambda^{k}(I)} p d x=2 \pi I_{j}, \quad j=1,2, \ldots, k, \\
(2 \pi)^{-1} \oint_{\gamma_{l}(I) \in \Lambda^{n}(I, \rho)} p d x=\rho_{l}, \quad l=1,2, \ldots, n-k .
\end{gathered}
$$

Обозначим индексы Маслова циклов $\Gamma_{j}$ и $\gamma_{l}(5.6)$ лагранжева тора $\Lambda^{n}$ через $M_{j}$ и $m_{l}$ соответственно. Имеем

$$
\operatorname{Ind} \Gamma_{j}=M_{j}=\left.\frac{1}{\pi} \operatorname{Arg} J(\varphi, \eta)\right|_{\varphi_{j}=\varphi_{j}^{0}} ^{\varphi_{j}=\varphi_{j}^{0}+2 \pi}, \quad j=1,2, \ldots, k,
$$

и

$$
\text { Ind } \gamma_{l}=m_{l}=\left.\frac{1}{\pi} \operatorname{Arg} J(\varphi, \eta)\right|_{\eta_{l}=\eta_{l}^{0}} ^{\eta_{l}=\eta_{l}^{0}+2 \pi}, \quad l=1,2, \ldots, n-k .
$$

Числа $\sigma_{j}(5.2)$ определены как для регулярного, так и нерегулярного комплексного ростка. В случае, если комплексный росток регулярньй, имеет место следующий факт.

\footnotetext{
${ }^{5}$ Здесь и всюду ниже в этом пункте зависимость величин от параметров $I$ и $\rho$ будем опускать.
} 
Теорема 2. Индексы регулярного комплексного ростка для ииклов $\Gamma_{j}(I), j=$ $1,2, \ldots, k$, на инвариантном изотропном торе $\Lambda^{k}(I)$ и индексы Маслова ииклов $\Gamma_{j}$ (5.8а) на почти инвариантном лагранжевом торе $\Lambda^{n}(I, \rho)$ (4.1) совпадают. Индекс Маслова ииклов $\gamma_{l}, l=1,2, \ldots, n-k$, на $\Lambda^{n}(I, \rho)$ равен $2\left(m_{l}=2\right.$, $l=1,2, \ldots, n-k)$.

ЗАмЕчАниЕ 4 . В полномерном случае индекс Маслова можно связать с числом пересечений пути $\Gamma_{j}(I)$ с циклом особенностей лагранжева многообразия $\Lambda^{n}$ относительно проектирования в конфигурационное пространство $\mathbb{R}_{x}^{n}$, при этом важно, что в общем положении размерность $\operatorname{dim} \Sigma=n-1$ (см. [53]). В неполномерном случае это не так (см. [54], [55]): в общем положении $\operatorname{dim} \Sigma \leqslant k-2$, так что путь $\Gamma_{j}$ можно выбрать таким образом, что он вообще не будет пересекать $\Sigma$. Более того, для многих примеров особенностей вообще нет (см. [13], [23]), а индекс Маслова комплексного ростка отличен от нуля. С другой стороны, если изотропный тор принадлежит инвариантному относительно системы (2.1) $2 k$-мерному симплектическому подпространству ${ }^{6}$, например для простоты евклидову $\mathbb{R}^{2 k}$, то в этом пространстве $k$-мерньй тор $\Lambda^{k}$ будет уже полномерным лагранжевьм многообразием и можно определить его индексы Маслова. С точки зрения матриц $B$ и $C$ это означает, что их можно представить в блочно-дигональном виде

$$
B=\left(\widetilde{B}, P_{\varphi}\right)=\left(\begin{array}{cc}
B_{1} & 0 \\
0 & \widetilde{P}_{\varphi}
\end{array}\right), \quad C=\left(\widetilde{C}, X_{\varphi}\right)=\left(\begin{array}{cc}
C_{1} & 0 \\
0 & \widetilde{X}_{\varphi}
\end{array}\right)
$$

где вещественные $(k \times k)$-матрищы $\widetilde{P}_{\varphi}, \widetilde{X}_{\varphi}$ составлены из ненулевых компонент касательных векторов к $\Lambda^{k}$, а якобиан $J$ можно представить в виде произведения $J_{1} J_{2}$, где $J_{1}=\operatorname{det}\left(C_{1}-i \varepsilon B_{1}\right), J_{2}=\operatorname{det}\left(\widetilde{X}_{\varphi}-i \varepsilon \widetilde{P}_{\varphi}\right)$. Полное приращение аргумента якобиана $J$ по циклу $\Gamma_{j}(I)$, деленное на $\pi$, тем самым складьвается из “обычного” индекса Маслова ${ }^{7}$ полномерного в $\mathbb{R}^{2 k}$ тора $\Lambda^{k}\left(M_{j}=\left.\frac{1}{\pi} \Delta \operatorname{Arg} J_{2}\right|_{\Gamma_{j}}\right)$ и "собственно индекса комплексного ростка" ( $\left.\widetilde{\sigma}_{j}=\left.\frac{1}{\pi} \Delta \operatorname{Arg} \operatorname{det} J_{1}\right|_{j}\right)$. Заметим, что в случае $k=1$ последний совпадает [23], [30]-[32], [58], [59] с индексом Гельфанда-Лидского линейных гамильтоновых систем с периодическими коэффициентами [60]. Тем самьм, на приращение аргумента "укороченного якобиана" $J_{1}$ можно смотреть как на аналог индекса Гельфанда-Лидского для линейных гамильтоновых систем с почти периодическими коэффициентами. Такое "расщепление" полного индекса комплексного ростка может оказаться удобным при решении конкретных задач, хотя конечно, не является обязательным. Наконец, в общей ситуации указанное "расщепление" полного индекса Маслова комплексного ростка едва ли представляется возможным даже в случае замкнутых траекторий.

5.4. Условие квантования Бора-Зоммерфельда-Маслова. Условия квантования гладкого семейства инвариантных изотропных торов $\Lambda^{k}(I)$ с комплексным ростком $r^{n}(I)$ есть условие квантования параметров семейства $I$, т.е. переменных действия $I=$ $\left(I_{1}, \ldots, I_{j}, \ldots, I_{k}\right) \in M_{I}$ на $\Lambda^{k}(I)$. Оно состоит в выборе при каждом фиксированном $h$, $0<h<h_{0}$, где $h_{0}$ достаточно мало, дискретного набора значений $I^{\nu}(h) \in M_{E}$ в соответствии с формулой

$$
I_{j}^{\nu}(h)=h\left(\nu_{j}+\frac{\sigma_{j}}{4}\right), \quad \nu_{j} \in \mathbb{Z}^{+}, \quad j=1,2, \ldots, k,
$$

\footnotetext{
${ }^{6}$ Такая ситуация типична для частично-интегрируемых гамильтоновых систем (см., например, $[56],[57],[12])$.

${ }^{7} \mathrm{~B}[30],[31]$ такой индекс называется тангенциальным.
} 
где $\sigma_{j}$ - индекс комплексного ростка цикла $\Gamma_{j}(I)$ на изотропном торе $\Lambda^{k}(I)$. Условие квантования (5.9) гарантирует построение канонического оператора на квантованном семействе изотропных торов $\Lambda^{k}(I)$ с комплексным ростком $r^{n}(I)$, т.е. при $I=I^{\nu}(h)$ (см. [13], [15], [16]). Квантование переменных действия на неполномерных $\Lambda^{k}(I)$ торах по форме не отличается от условий квантования (1.1) полномерных торов, но в отличие от последнего случая из-за "потери размерности" $(k<n)$ его недостаточно для получения квазиклассической асимптотики спектральных серий исходного оператора. Для этого необходимы характеристики динамического потока $g_{H}^{t}$ и его линеаризации, порождаемые свойствами инвариантности изотропного тора и комплексного ростка ${ }^{8}$.

\section{6. Динамические характеристики инвариантных изотропных торов с} комплексным ростком и квазиклассические энергетические уровни.

6.1. Сильно нерезонансный случай. Сначала рассмотрим ситуацию, когда выполнено условие сильной нерезонансности [34], [35], [48], [51]:

УСловиЕ d). Частоты условно-периодического движения $\omega_{j}(I), j=1,2, \ldots, k$, на торе $\Lambda^{k}(I)$ при $I=I^{\nu}(h)$ из (5.9) диофантово несоизмеримы:

$$
\langle\omega(I), m\rangle \geqslant C|m|^{-r}, \quad C>0, \quad r>0, \quad m \in \mathbb{Z}^{k}
$$

\section{Определим динамическую характеристику “квазивакуумного” состояния}

$$
\lambda_{0}\left(I^{\nu}(h)\right)=\left.\lim _{t \rightarrow \infty} \frac{1}{t} \int_{0}^{t} \operatorname{tr} A(\omega t+\varphi, I)\right|_{I=I^{\nu}(h)} d t=\frac{1}{(2 \pi)^{k}} \int_{\Lambda^{k}\left(I^{\nu}(h)\right)} \operatorname{tr} A\left(\varphi, I^{\nu}(h)\right) d \varphi
$$

где матрица $A(\omega t+\varphi, I)$ - матрица системы в вариациях на $\tilde{r}^{n-k}$ - определена в $(3.5)$.

Нормальная форма гамильтониана в окрестности изотропного тора с комплексным ростком порождает динамический поток $G_{t}: \Lambda^{k}(I) \times \mathbb{C}^{n-k} \rightarrow \Lambda^{k}(I) \times \mathbb{C}^{n-k}$, определяемьй сдвигами вдоль траекторий следующей системы:

$$
\begin{gathered}
\dot{\varphi}=\omega(I) \rightarrow \varphi_{t}(\varphi)=\omega(I) t+\varphi, \quad \varphi \in \Lambda^{k}(I), \\
\dot{\bar{\xi}}=-i A(\omega t+\varphi, I) \bar{\xi}, \quad \bar{\xi}(t, \varphi) \in \mathbb{C}^{n-k}
\end{gathered}
$$

Обозначим через $\mathscr{H}$ гильбертово пространство функций, заданных на торе, со значениями в пространстве $F_{2}, \mathscr{H}=L_{2}\left(\Lambda^{k}, F_{2}\right)$, где $F_{2}$ - фоковское пространство целых антианалитических функций $g(\bar{\xi}), \xi \in \mathbb{C}^{n-k}$, для которых конечна норма

$$
\|g\|^{2}=\int|g(\bar{\xi})|^{2} e^{-|\xi|^{2}} d \xi d \bar{\xi}
$$

где $d \xi d \bar{\xi}=\frac{1}{\pi} d \xi^{\prime} d \xi^{\prime \prime}\left(\xi^{\prime}=\operatorname{Re} \xi, \xi^{\prime}=\operatorname{Im} \xi^{\prime \prime}\right)$ - нормированная мера Лебега на $\mathbb{C}^{n-k}$ (см., например, [61]). Таким образом, для элемента $f \in \mathscr{H}$ его норма

$$
\|f\|_{\mathscr{H}}^{2}=\frac{1}{(2 \pi)^{k}} \int_{\Lambda^{k}} \int_{\mathbb{C}^{n-k}}|f(\varphi, \bar{\xi})|^{2} e^{-|\xi|^{2}} d \xi d \bar{\xi} d \varphi
$$

\footnotetext{
${ }^{8}$ В полномерном интегрируемом случае все эти характеристики легко находятся из представления гамильтониана в переменных действия $H=H(I)$.
} 
Очевидно, нормированная мера

$$
d \mu=\frac{e^{-|\xi|^{2}}}{(2 \pi)^{k}} d \xi d \bar{\xi} d \varphi
$$

инвариантна относительно сдвигов вдоль системы (6.3).

Рассмотрим в пространстве $\mathscr{H}$ (с инвариантной мерой) спектральную задачу [62] для отображения $G_{t}^{*}: \mathscr{H} \rightarrow \mathscr{H}$, индуцированного потоком $G_{t}$,

$$
G_{t}^{*} f_{\lambda}(\varphi, \bar{\xi})=e^{i \lambda t} f_{\lambda}(\varphi, \bar{\xi}), \quad f_{\lambda} \in \mathscr{H}, \quad \lambda \in \mathbb{R}
$$

где, напомним, $\left(G_{t}^{*} f_{\lambda}\right)(\varphi, \bar{\xi})=f_{\lambda}(\omega(I) t+\varphi, \bar{\xi}(t, \varphi))$ и $\bar{\xi}(0, \varphi)=\bar{\xi}$.

Tеорема 3. Пусть существует собственная функиия $f_{\lambda}(\varphi, \xi)$ оператора $G_{t}^{*}$ (собственная функиия потока $G_{t}(6.2)$ ), отвечающая собственному значению $\lambda=$ $\lambda(I)$. Тогда әто число совместно с $\lambda_{0}\left(I^{\nu}(h)\right)$ определяет асимптотику при $h \rightarrow 0$ собственного значения исходной квантовой задачи

$$
E_{\nu}^{\lambda}(h)=H_{0}\left(I^{\nu}(h)\right)+h\left(\frac{\lambda_{0}\left(I^{\nu}(h)\right)}{2}+\lambda\left(I^{\nu}(h)\right)\right)+O\left(h^{2}\right),
$$

где числа $I_{j}^{\nu}(h), j=1,2, \ldots, k$, определены условием квантования $(6.1), H_{0}(I)=$ $\left.H\right|_{\Lambda^{k}(I)}$.

ЗАмЕчАнИЕ 5. Ясно, что по крайней мере функция $f=1$ и число $\lambda=0$ являются решением задачи (6.4), именно им соответствует "квазивакуумная" квазимода исходного оператора. Кроме того, очевидно, что если $f, \lambda$-решение задачи (6.4), то и для любого $k$-мерного вектора $l$ с целочисленными компонентами пара $f e^{i\langle l \cdot \varphi\rangle}, \lambda+\langle\omega, l\rangle-$ тоже решение. Такие новые решения не приводят к новым асимптотическим собственным функциям и значениям, а лишь к их перенумерации: $\nu \rightarrow \nu+l$ (см. [23]).

6.2. Асимптотика спектральных серий, отвечаюших регулярному комплексному ростку, и сравнение с правилом квантования для полномерного случая. Рассмотрим случай регулярного комплексного ростка, т.е. случай, когда матрица $i A(\omega(I) t+\varphi, I)$ в системе (6.4) приводится к диагональной матрице $\hat{\beta}(I)=\left(\beta_{l}(I)\right), l=1,2, \ldots, n-k, \mathrm{c}$ помощью унитарной матрицы $\widetilde{U}_{0}(\omega(I) t+\varphi)$. Иначе говоря, в этом случае можно считать, что в подходящих координатах матрица $i A$ в $(3.5)$ диагональна и равна $\operatorname{diag}\left(\beta_{j}\right)$, $j=1, \ldots, k$. Тогда, очевидно, число

$$
\lambda(I)=\sum_{l=1}^{n-k} \beta_{l}(I) \mu_{l}, \quad \mu_{l} \in \mathbb{Z}^{+}
$$

является собственным значением задачи (6.4), отвечающим собственной функции $f_{\lambda}(\varphi$, $\bar{\xi})$ - однородному полиному степени $|\mu|=\mu_{1}+\mu_{2}+\cdots+\mu_{n-k}$ вида

$$
f_{\lambda}(\varphi, \bar{\xi})=\left(\widetilde{U}_{0}^{*}(\varphi) \bar{\xi}\right)^{\mu}, \quad \mu \in \mathbb{Z}_{+}^{n-k}
$$


Здесь и ниже через $q^{\mu}, q \in \mathbb{C}^{n-k}$, обозначен полином $q_{1}^{\mu_{1}} q_{2}^{\mu_{2}} \cdots q_{n-k}^{\mu_{n-k}}$. Динамическая характеристика $\lambda_{0}$ “квазивакуумного" состояния (6.3) на квантованном семействе изотропных торов $\Lambda^{k}(I)$ с регулярным комплексным ростком имеет вид

$$
\lambda_{0}\left(I^{\nu}(h)\right)=\sum_{l=1}^{n-k} \beta_{l}\left(I^{\nu}(h)\right)
$$

Окончательно, из (6.5)-(6.8) найдем серию [15], [16] асимптотических собственных значений ${ }^{9}$ исходного оператора $\widehat{H}$, отвечающую семейству $\Lambda^{k}\left(I^{\nu}(h)\right), r^{n}\left(I^{\nu}(h)\right)$ :

$$
E_{\nu, \mu}(h)=H_{0}\left(I^{\nu}(h)\right)+h \sum_{l=1}^{n-k} \beta_{l}\left(I^{\nu}(h)\right)\left(\mu_{l}+1 / 2\right)+O\left(h^{2}\right),
$$

где

$$
I^{\nu}(h)=\left(I_{1}^{\nu}(h), \ldots, I_{k}^{\nu}(h)\right), \quad I_{j}^{\nu}(h)=h\left(\nu_{j}+\frac{\sigma_{j}}{4}\right), \quad j=1, \ldots, k, \quad \nu_{j} \in \mathbb{Z}^{+} .
$$

Сравним полученньй результат с асимптотикой спектра $E_{\nu}(h)$ оператора $\widehat{H}$, которую мы получаем с помощью правила квантования Бора-Зоммерфельда (с учетом индекса Маслова) для $n$-мерных лагранжевых почти инвариантных торов $\Lambda^{n}(I, \rho)(4.1)$. А именно, из формулы (6.2) и вида гамильтониана $H$ в нормальной форме (см. теорему 1 , (3.4)) имеем

$$
E_{\nu}(h)=H_{0}\left(I^{\nu}(h)\right)+h \sum_{l=1}^{n-k}\left(\nu_{k+l}+\frac{m_{k+l}}{4}\right) \beta_{l}\left(I^{\nu}(h)\right)+O\left(h^{3 / 2}\right),
$$

где

$$
I^{\nu}(h)=\left(I_{j}^{\nu}(h)\right), \quad j=1,2, \ldots, k, \quad I_{j}^{\nu}(h)=h\left(\nu_{j}+\frac{M_{j}}{4}\right), \quad \nu_{j} \in \mathbb{Z}^{+} .
$$

Если теперь воспользоваться результатом теоремы 2, то из сравнения формул (6.9)(6.10) и (6.11)-(6.12) приходим немедленно к следующему результату (ср. с подходом работы [63] для случая замкнутых траекторий).

Теорема 4. Пусть выполнены условия теоремы 1 и условие d), и пусть система в вариациях (3.6) является приводимой. Тогда серии асимптотических собственных значений исходного оператора $\widehat{H}$, отвечающие проквантованным семействам изотропных торов с регулярным комплексным ростком (в силу правила квантования (6.9)) и отвечающие семействам лагранжевых почти инвариантных торов $\Lambda^{n}(I, \rho)(4.1)$ (в силу условий квантования Бора-Зоммерфельда-Маслова (6.10)) совпадают с точностью до $O\left(h^{3 / 2}\right), h \rightarrow 0$.

\footnotetext{
${ }^{9}$ Как частный случай $k=1$ эта формула включает в себя формулы [13], [17], [19], [21], отвечающие замкнутым траекториям (замкнутым геодезическим).
} 
ЗАмечАниЕ 6. Тем самьм, формула (6.11) может быть получена предельным переходом из полномерного случая и тогда $\beta_{j}$ - "предельные осцилляторные" частоты. Такой предельный переход не имеет места для асимптотических собственных функций, отвечающих изотропньм торам с комплексньм ростком: их структура совершенно отлична от структуры асимптотических собственных функций в полномерной ситуации (см. [13], [15], [16]).

6.3. Спектральные серии в нерегулярном случае. В нерегулярном случае, когда система (3.6) с квазипериодическими коэффициентами неприводима (которьй, повидимому, можно назвать также слабохаотическим), решение спектральной задачи (6.4) (в некоторой области спектра) может быть сведено к решению спектральной задачи на изотропном торе $\Lambda^{k}$ для линейной системы дифференциальных уравнений в частных производных первого порядка относительно тензорного поля на торе $\left\{C_{\alpha}(\varphi)\right\}, \alpha=\left(\alpha_{1}, \ldots\right.$, $\left.\alpha_{n-k}\right),|\alpha|=L$, ранга $L$ :

$$
\begin{aligned}
& i \dot{C}_{\alpha}^{\lambda}(\varphi)-\left(\sum_{j=1}^{n-k} \alpha_{j} A_{j j}\right) C_{\alpha}^{\lambda}(\varphi) \\
& \quad-\sum_{j=1}^{n-k}\left(\alpha_{j}+1\right) \sum_{\substack{m=1 \\
m \neq j}}^{n-k} A_{j m} C_{\alpha_{1}, \ldots, \alpha_{j}+1, \ldots, \alpha_{m}-1, \ldots, \alpha_{n-k}}^{\lambda}=\lambda C_{\alpha}^{\lambda}(\varphi) .
\end{aligned}
$$

Здесь точка означает дифференцирование

$$
\frac{d}{d t}=\omega_{1} \frac{\partial}{\partial \varphi_{1}}+\cdots+\omega_{k} \frac{\partial}{\partial \varphi_{k}}
$$

и мы считаем, что $C_{\alpha_{1}, \ldots, L+1, \ldots,-1, \ldots, \alpha_{n-k}}^{\lambda}=0$. Система (6.13) имеет "ленточную" структуру: в уравнение с номером $\alpha$ (т.е. в уравнение, содержащее прозводную $\dot{C}_{\alpha}^{\lambda}$ ) входят только элементы с номером $\alpha$ и соседними с ним номерами. Например, в случае $n=4$ и $k=2$ система (6.13) имеет вид

$$
\begin{gathered}
i \dot{C}_{\alpha_{1}, \alpha_{2}}^{\lambda}-\left(\lambda+\alpha_{1} A_{11}+\alpha_{2} A_{22}\right) C_{\alpha_{1}, \alpha_{2}}^{\lambda} \\
=\left(\alpha_{1}+1\right) A_{12} C_{\alpha_{1}+1, \alpha_{2}-1}+\left(\alpha_{2}+1\right) A_{21} C_{\alpha_{1}-1, \alpha_{2}+1} \\
\quad \alpha_{1}, \alpha_{2} \in \mathbb{Z}^{+}, \quad C_{-1, L+1}=0, \quad C_{L+1,1}=0
\end{gathered}
$$

Теорема 5. Пусть выполнены условия теоремы 1 и условие d) (6.1), и пусть тензорное поле $C_{\alpha}^{\lambda}$ есть решение задачи (6.13) с собственным числом $\lambda$. Тогда әто число совместно с $\lambda_{0}\left(I^{\alpha}(h)\right)$ определяет по формуле (6.5) асимптотику при $h \rightarrow 0$ собственного значения исходной квантовой задачи. При этом соответствующая собственная функиия из $\mathscr{H}$ потока $G_{t}$ есть однородный полином аргумента $\bar{\xi}$ :

$$
f_{\lambda}(\varphi, \bar{\xi})=\sum_{|\alpha|=L} C_{\alpha}^{\lambda}(\varphi) \frac{\bar{\xi}^{\alpha}}{\sqrt{\alpha !}}, \quad \alpha \in\left(\mathbb{Z}^{+}\right)^{n-k}, \quad \xi \in \mathbb{C}^{n-k}
$$


Если собственное число $\lambda$ вырождено, то любая собственная функиия из भ̈ потока $G_{t}$ может быть представлена в виде линейной комбиначии функций (6.15) (конечной или бесконечномерной), возможно разной степени однородности $L$.

Нетрудно убедиться, что системы (2.2), (3.6) и (6.13) приводимы или неприводимы одновременно (поскольку диагональность матрицы системы (3.6) влечет диагональность матрицы системы (6.13), и наоборот). Поэтому теорема 4 вытекает из теоремы 3 : в координатах, в которых матрица системы (6.14) диагональна, ее решения суть тензорные поля $C_{\alpha}(\varphi)=\delta_{\alpha, \mu}, \lambda=\langle\beta, \mu\rangle$ и соответствущие однородные полиномы $f_{\lambda}$ суть мономы $\bar{\xi}^{\mu}$. Вопрос о существовании решений системы (6.13) в неприводимом случае является открытьм. Также не ясно, сушествуют ли решения спектральной задачи (6.4) в пространствах функций, отличных от $\mathscr{H}$, и если существуют, то можно ли их использовать для построения асимптотических собственных функций исходной задачи. Ответы на эти вопросы важны для понимания структуры спектра в "слабохаотических окрестностях" неполномерных изотропных торов. Заметим, что как система (3.6), так и система (6.13) могут разбиваться на блоки (“эргодические компоненты”), каждый из которых порождает свою часть в спектральной серии; предельные случаи - регулярный (приводимьй) и "полностью нерегулярный". Подчеркнем еще раз, что "квазивакуумная" квазимода при выполнении условия d) (6.1) существует всегда.

6.4. Слаборезонансный и резонансный случаи. Если частоты $\omega_{j}$ соизмеримы или плохо несоизмеримы (т.е. условие d) не выполнено), то вместо (6.4) целесообразно рассмотреть более общую задачу для функции $F_{\tilde{\lambda}}$ и числа $\tilde{\lambda}$ :

$$
G_{t}^{*} F_{\tilde{\lambda}}(\varphi, \bar{\xi})=e^{i \tilde{\lambda} t} \exp \left(-\frac{i}{2} \int_{0}^{t} \operatorname{tr} A(\omega(I) \tau+\varphi) d \tau\right) F_{\tilde{\lambda}}(\varphi, \bar{\xi}), \quad F_{\tilde{\lambda}} \in \mathscr{H}, \quad \tilde{\lambda} \in \mathbb{R} .
$$

При этом требуется ${ }^{10}$, чтобы $\tilde{\lambda}$ не зависело от углов $\varphi$. Тогда вместо формулы (6.5) для асимптотики собственных значений исходной задачи следует использовать формулу

$$
E_{\nu}^{\tilde{\lambda}}(h)=H_{0}\left(I^{\nu}(h)\right)+h \tilde{\lambda}\left(I^{\nu}(h)\right)+O\left(h^{2}\right) .
$$

Нетрудно показать, что при вьполнении условия d) задача (6.16) редуцируется к задаче (6.4) и формула (6.17) переходит в формулу (6.5).

Резонансная ситуация обсуждалась с разных точек зрения в работах [23]-[26], [29], [31]. Отметим, что в плохо несоизмеримом случае повидимому возможна противоположная п. 6.1 ситуация, когда "квазивакуумное" состояние не существует, а "возбужденные" состояния существуют. С точки зрения нормальных форм эта ситуация характеризуется тем обстоятельством, что число переменных действия в главной части гамильтониана $H$ становится меньше $k$. Тогда торы $\Lambda^{k}$ определяются уже квадратичной частью и, тем самым, могут (сильно) зависеть от номера $\mu$ "возбужденной" квазимоды (см. формулу (6.6)). Вообще в классической механике такая ситуация изучена значительно слабее, чем сильнорезонансная. С другой стороны, с точки зрения квантовой механики эта ситуация представляет не меньший интерес ${ }^{11}$.

\footnotetext{
${ }^{10} \mathrm{~B}$ случае несоизмеримых частот это требование всегда выполнено; в случае соизмеримых частот оно приводит к отбору пригодных для квантования семейств торов $\Lambda^{k}(I)$, см. [23]-[26], [29], [31] .

${ }^{11} \mathrm{~B}$ том числе и с точки зрения численной реализации изложенной схемы квантования.
} 


\section{7. Индивидуальные инвариантные изотропные торы с комплексным рост-} ком: возмущение и квантование. Рассуждения пп. 3-6 несправедливы, если семейство торов $\Lambda^{k}$ параметризуется числом параметров, меньшим чем их размерность $k$, в частности, если $\Lambda^{k}$ - индивидуальньй $k$-мерньй тор $\Lambda^{k}\left(I^{0}\right)$. Тем не менее, при условии, что движение на торе $\Lambda^{k}\left(I^{0}\right)$ условно периодическое с частотами $\omega\left(I^{0}\right)$ и что существует комплексньй росток $r^{n}\left(I^{0}\right)$ на $\Lambda^{k}\left(I^{0}\right)$, может быть построено семейство почти инвариантных изотропных $k$-мерных торов $\Lambda^{k}\left(I^{0}+\Delta I\right)$, зависящих от $k$ параметров $\Delta I=\left(\Delta I_{1}, \ldots, \Delta I_{k}\right)$, которые являются "отклонениями" переменных действия от действия $I^{0}$ на торе $\Lambda^{k}\left(I^{0}\right)$. Для нужд квазиклассического квантования по $\bmod O\left(h^{2}\right)$ комплексньй росток может быть выбран не зависяшим от $\Delta I$ и совпадающим с комплексным ростком $r^{n}\left(I^{0}\right)$. Не вдаваясь в детали, кратко обсудим соответствующие конструкции.

Аналогично п. 3 , в окрестности тора $\Lambda^{k}\left(I^{0}\right)$ введем симплектические координаты $y^{\prime}=\left(I=I^{0}=\Delta I, \varphi, \xi, \bar{\xi}\right)$, полагая

$$
y=y\left(y^{\prime}\right)=Y\left(\varphi, I^{0}\right)+\frac{1}{2}\left(V\left(\varphi, I^{0}\right) \xi+\bar{V}\left(\varphi, I^{0}\right) \bar{\xi}\right)+Y_{I}\left(\varphi, I^{0}\right)(\Delta I+g(\varphi, I, \xi, \bar{\xi}))
$$

Здесь вектор-функция $Y$, комплексная $((2 n) \times(n-k))$-матрица $V$ и $g-k$-мерная вектор-функция - те же, что и в $(3.1),(3.2)$, а под $Y_{I}\left(\varphi, I^{0}\right)$ мы понимаем $((2 n) \times k)$-матрицу (cp. с [33]) вида

$$
Y_{I}\left(\varphi, I^{0}\right)=Y_{\varphi} Q+\left(\frac{i}{2} \bar{V} \bar{V}^{*}-\frac{i}{2} V V^{*}+J\right) Y_{\varphi} M
$$

Здесь аргументы у всех матрищ в правой части $-\varphi, I^{0} ; M=\left({ }^{t} Y_{\varphi} Y_{\varphi}\right)^{-1}$, a $(k \times k)$-гладкая матрица $Q(\varphi)$ выбирается (в рассматриваемом приближении) произвольньм образом, например, можно считать $Q=0$.

Тогда формула $y=Y\left(\varphi, I^{0}\right)+Y_{I}\left(\varphi, I^{0}\right)$ определяет почти инвариантные $\bmod$ $O\left((\Delta I)^{2}\right)$-почти изотропные (в смысле приближенного $\bmod O\left((\Delta I)^{2}\right)$-равенства нулю скобок Лагранжа) торы $\Lambda^{k}\left(I^{0}+\Delta I\right)$ с условно периодическим движением с частотами $\omega\left(I^{0}\right)$. Комплескньй росток $r^{n}\left(I^{0}\right)$ на этих торах будет почти инвариантньг в том смысле, что соответствующие функции удовлетворяют системе в вариациях по mod $O(\Delta I)$. Нормальная форма гамильтониана $H$ в окрестности тора $\Lambda^{k}\left(I^{0}\right)$ имеет вид

$$
H(y)=\left.H\right|_{\Lambda^{k}\left(I^{0}\right)}+\left\langle\omega\left(I^{0}\right), \Delta I\right\rangle+\frac{1}{2}\left\langle\bar{\xi}, A\left(\varphi, I^{0}\right) \xi\right\rangle+O\left(\|\xi\|^{3}+\|\Delta I\|\|\xi\|^{2}+\|\Delta I\|^{2}\right) .
$$

Наконец, действия на семействе построенных торов равны

$$
\frac{1}{2 \pi} \oint_{\Gamma_{l}} p d q=I_{l}^{0}+\Delta I_{l}, \quad l=1, \ldots, k
$$

Теперь мы можем применить общую схему квантования семейства из пш. 4-6 к построенному семейству почти инвариантных торов с комплексным ростком. При этом следует считать, что числа $\nu$ таковы, что соответствующие им проквантованные параметры $\Delta I^{\nu}(h)$ имеют по крайней мере порядок $O\left(h^{3 / 4}\right)$. В формулах и уравнениях (6.5)-(6.8), (6.15)-(6.17) $I^{\nu}$ без потери точности можно заменить на $I^{0}$, а в формулах $(6.9),(6.11)$ следует положить

$$
H_{0}\left(I^{\nu}\right)=H_{0}\left(I^{0}\right)+\left\langle\omega\left(I^{0}\right), \Delta I^{\nu}\right\rangle, \quad \Delta I^{\nu}=h\left(\nu_{j}+\frac{\sigma_{j}}{4}\right)-I_{j}^{0}(h), \quad j=1, \ldots, k, \quad \nu_{j} \in \mathbb{Z}^{+} .
$$


ЗАмЕчАниЕ 7. Введем матрицу

$$
R=M^{t} Y_{\varphi}\left(J\left[H_{y y}, J\right] Y_{\varphi} M-\bar{V} \dot{G}-V \dot{\bar{G}}\right)
$$

размерности $k \times k$, где $((n-k) \times k)$-матрица $G=i^{t} V Y_{\varphi} M / 2$. Предположим, что среднее

$$
\frac{\partial \omega}{\partial I}=\lim _{t \rightarrow \infty} \frac{1}{t} \int_{0}^{t} R(\varphi+\omega \tau) d \tau
$$

не зависит от $\varphi$. (Это всегда так для несоизмеримых частот.) Предположим, что матрица $Q(\varphi+\omega t)$ размерности $k \times k$ на торе $\Lambda^{k}\left(I^{0}\right)$ удовлетворяет гомологическому уравнению

$$
Q(\varphi+\omega t)=Q(\varphi)+\int_{0}^{t} R(\varphi+\omega \tau) d \tau
$$

Тогда можно уточнить каноническое ${ }^{12}$ преобразование таким образом, что нормальная форма гамильтониана $H$ примет вид

$$
\begin{aligned}
H(y)= & \left.H\right|_{\Lambda^{k}\left(I^{0}\right)}+\left\langle\omega\left(I^{0}\right), \Delta I\right\rangle+\frac{1}{2}\left\langle\Delta I, \frac{\partial \omega}{\partial I} \Delta I\right\rangle+\frac{1}{2}\left\langle\bar{\xi}, A\left(\varphi, I^{0}\right) \xi\right\rangle \\
& +O\left(\|\xi\|^{3}+\|\Delta I\|\|\xi\|^{2}+\|\Delta I\|^{3}\right) .
\end{aligned}
$$

Это позволяет расширить область изменения чисел $\nu$ до значений $\Delta I^{\nu} \approx \sqrt{h}$ и иногда частично снять асимптотическое вырождение спектра (если оно имеет место). Для получения ответа о снятии вырождения в приближении $O\left(h^{5 / 2}\right)$ при $\Delta I^{\nu} \approx h$ нужно (по крайней мере) построить нормальную форму с точностью до $O(\|\xi\|+\sqrt{\|\Delta I\|})^{5}$.

\section{8. Доказательства утверждениий пп. 2-7.}

ДокАЗАТЕЛЬСтво ЛЕммы 1 . Пусть векторы $R_{1}(\varphi), R_{2}(\varphi), \ldots, R_{2 n-2 k}(\varphi)$ образуют симплектический базис в слое нормального расслоения $N\left(\Lambda^{k}\right)$ над точкой $\varphi \in \Lambda^{k}$. В силу тривиальности расслоения $E\left(\Lambda^{k}\right)$ базис в слое $E_{\varphi}\left(\Lambda^{k}\right), \varphi \in \Lambda^{k}$, составленньй из этих векторов и касательных векторов $Y_{\varphi_{1}}(\varphi), Y_{\varphi_{2}}(\varphi), \ldots, Y_{\varphi_{k}}(\varphi)$, можно дополнить векторами $Y_{1}(\varphi), Y_{2}(\varphi), \ldots, Y_{k}(\varphi)$, заданными на торе $\Lambda^{k}$, до симплектического базиса в ${ }^{\mathbb{C}} T_{\varphi} \mathbb{R}_{p, x}^{2 n}, \varphi \in \Lambda^{k}$. Матрищы $R(\varphi), Y_{\varphi}(\varphi), Y(\varphi)$, составленные из соответствующих векторов, заданные на торе $\Lambda^{k}$, удовлетворяют условиям:

1) ${ }^{t} R J Y_{\varphi}=0,{ }^{t} R J Y=0$;

2) ${ }^{t} Y J Y_{\varphi}=E_{k}$

3) ${ }^{t} R J R=E_{2 n-2 k}$.

Пусть теперь существует комплексный росток $r^{n}$ на $\Lambda^{k}$. Тогда любой вектор $\xi$ на плоскости $r^{n}(\varphi), \varphi \in \Lambda^{k}$, задается с помощью равенства

$$
\xi=R(\varphi) M \alpha^{\prime}+Y_{\varphi} \alpha^{\prime \prime}, \quad \alpha^{\prime} \in \mathbb{C}^{n-k}, \quad \alpha^{\prime \prime} \in \mathbb{C}^{k}
$$

12Это уточнение опирается на результаты работы [49]. Пользуясь случаем, автор работы [49] высказывает сожаление, что он не знал о близких результатах, полученных ранее в работах [64], [65]. 
г де $\left(\alpha^{\prime}, \alpha^{\prime \prime}\right)$ - координаты на плоскости $r^{n}(\varphi), M-((2 n-2 k) \times(n-k))$-матрица, заданная на универсальной накрьвающей $\widetilde{\Lambda}^{k}$, которую мы представим в блочном виде

$$
M=\left(\begin{array}{l}
\widetilde{B} \\
\widetilde{C}
\end{array}\right)
$$

$\widetilde{B}$ и $\widetilde{C}$ - невырожденные $((n-k) \times(n-k))$-матрицы, определенные на $\widetilde{\Lambda}^{k}$. Обозначим через $B$ и $C(n \times n)$-матрицы (заданные на $\left.\widetilde{\Lambda}^{k}\right)$, определяемые равенством

$$
\left(\begin{array}{l}
B \\
C
\end{array}\right)=\left(R(\varphi) M, Y_{\varphi}(\varphi)\right)
$$

В силу определения комплексного ростка условия лагранжевости и диссипативности плоскости $r^{n}(\varphi), \varphi \in \Lambda^{k}$, означают, что матрицы $B$ и $C$ обладают свойствами

$$
{ }^{t} C B-{ }^{t} B C=0
$$

И

$$
\frac{1}{2 i}\left(C^{*} B-B^{*} C\right) \geqslant 0 .
$$

Из определения матриц $B$ и $C$ и условий нормировки 1)-3) симплектического базиса ${ }_{\text {в }}{ }^{C} T_{\varphi} \mathbb{R}_{p, x}^{2 n}$ имеем равенство

$$
\frac{1}{2 i}\left(C^{*} B-B^{*} C\right)=\left(\begin{array}{cc}
\widetilde{C}^{*} \widetilde{B}-\widetilde{B}^{*} \widetilde{C} & 0 \\
0 & 0
\end{array}\right),
$$

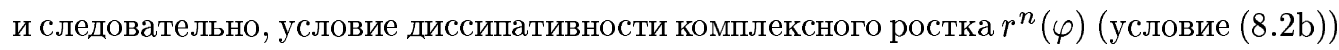
означает, что $((n-k) \times(n-k))$-матрица

$$
\widetilde{\mathscr{E}}=\frac{1}{2 i}\left(\widetilde{C}^{*} \widetilde{B}-\widetilde{B}^{*} \widetilde{C}\right)>0 .
$$

Значит, можно определить матрищы $\widetilde{\mathscr{E}}^{1 / 2}$ и $\widetilde{\mathscr{E}}^{-1 / 2}$. Перейдем на плоскости $\tilde{r}^{n-k}(\varphi) \mathrm{k}$ новому базису, положив $\widetilde{M}=M \widetilde{\mathscr{E}}^{-1 / 2}$. Тогда матрицу $\widetilde{\mathscr{E}}$ в $(8.3)$ можно считать единичной матрищей $E_{n-k}$ и условие диссипативности ростка примет вид

$$
\widetilde{C}^{*} \widetilde{B}-\widetilde{B}^{*} \widetilde{C}=2 i E_{n-k}
$$

Рассмотрим теперь $((n-k) \times(n-k))$-матрицу $\widetilde{C} \widetilde{C}^{*}=\Gamma$. Очевидно, что эрмитова матрица $\Gamma$ положительна. Покажем, что $\Gamma$ задана на торе $\Lambda^{k}$. Пусть $\varphi \in \mathbb{R}^{k}-$ координаты на универсальной накрывающей $\widetilde{\Lambda}^{k}$ относительно стандартного базиса $e_{j}$, $j=1,2, \ldots, k$. Для матриц $\widetilde{B}$ и $\widetilde{C}$, заданных на $\widetilde{\Lambda}^{k}$, имеем $\widetilde{B}\left(\varphi+2 \pi e_{j}\right)=\widetilde{B}(\varphi) U_{j}$, $\widetilde{C}\left(\varphi+2 \pi e_{j}\right)=\widetilde{C}(\varphi) U_{j}$, где $U_{j}-$ невырожденная $((n-k) \times(n-k))$-матрища. В силу условия диссипативности (8.4) $U_{j}$ - унитарная матрица. Действительно, имеем

$$
\begin{aligned}
& \widetilde{C}^{*}\left(\varphi+2 \pi e_{j}\right) \widetilde{B}\left(\varphi+2 \pi e_{j}\right)-\widetilde{B}^{*}\left(\varphi+2 \pi e_{j}\right) \widetilde{C}\left(\varphi+2 \pi e_{j}\right) \\
& \quad=U^{*}\left(\widetilde{C}^{*}(\varphi) \widetilde{B}(\varphi)-\widetilde{B}^{*}(\varphi) \widetilde{C}(\varphi)\right) U=2 i U^{*} U=2 i E_{n-k}
\end{aligned}
$$


Поэтому матрица $\Gamma(\varphi) 2 \pi$-периодическая по каждому своему аргументу $\left(\varphi_{1}, \varphi_{2}, \ldots\right.$, $\left.\varphi_{k}\right)$, так как

$$
\Gamma\left(\varphi+2 \pi e_{j}\right)=\widetilde{C}^{*}\left(\varphi+2 \pi e_{j}\right) \widetilde{C}\left(\varphi+2 \pi e_{j}\right)=\widetilde{C}(\varphi) U_{j} U_{j}^{*} \widetilde{C}^{*}(\varphi)=\widetilde{C}(\varphi) \widetilde{C}^{*}(\varphi)=\Gamma(\varphi) .
$$

Теперь представим матрицу $\widetilde{C}$ в виде $\widetilde{C}=\sqrt{\widetilde{C} \widetilde{C}^{*}} \Phi$ ("полярное разложение", см. [66, гл. 9]), где $\Phi=\left(\widetilde{C} \widetilde{C}^{*}\right)^{-1 / 2} \widetilde{C}(\operatorname{det} \Phi=1)$. Перейдем в плоскости фактора комплексного ростка $\tilde{r}^{n-k}(\varphi)$ к новому базису, сделав замену координат $M^{\prime}=M \Phi^{-1}$; тогда в новых координатах получим матрицу

$$
\left(\begin{array}{c}
\widetilde{B}^{\prime} \\
\widetilde{C}^{\prime}
\end{array}\right)=\left(\begin{array}{c}
\widetilde{B} \\
\widetilde{C}
\end{array}\right) \Phi^{-1}
$$

которая является на торе $\Lambda^{k}$ матрицей, $2 \pi$-периодической по каждому своему аргументу $\left(\varphi_{1}, \varphi_{2}, \ldots, \varphi_{k}\right)=\varphi \in \mathbb{R}^{k}$. Действительно, имеем:

$$
\begin{aligned}
& \left(\begin{array}{c}
\widetilde{B}^{\prime} \\
\widetilde{C}^{\prime}
\end{array}\right)\left(\varphi+2 \pi e_{j}\right)=\left(\begin{array}{c}
\widetilde{B}\left(\varphi+2 \pi e^{j}\right) \Phi^{-1}\left(\varphi+2 \pi e_{j}\right) \\
\widetilde{C}\left(\varphi+2 \pi e_{j}\right) \Phi^{-1}\left(\varphi+2 \pi e_{j}\right)
\end{array}\right) \\
& \quad=\left(\begin{array}{c}
\widetilde{B}\left(\varphi+2 \pi e_{j}\right) \Phi^{-1}\left(\varphi+2 \pi e_{j}\right) \\
\widetilde{C} \widetilde{C}^{*}\left(\varphi+2 \pi e_{j}\right)
\end{array}\right)=\left(\begin{array}{c}
\widetilde{B}\left(\varphi+2 \pi e_{j}\right) \Phi^{-1}\left(\varphi+2 \pi e_{j}\right) \\
\widetilde{C} \widetilde{C}^{*}(\varphi)
\end{array}\right) \\
& \quad=\left(\begin{array}{c}
\widetilde{B}\left(\varphi U_{j} \widetilde{C}^{-1}\left(\varphi+2 \pi e_{j}\right)\right)\left(\widetilde{C} \widetilde{C}^{*}\right)^{1 / 2}(\varphi) \\
\left(\widetilde{C} \widetilde{C}^{*}\right)^{1 / 2}(\varphi)
\end{array}\right)=\left(\begin{array}{c}
\widetilde{B}\left(\varphi U_{j} \widetilde{C}^{-1}(\varphi)\right)\left(\widetilde{C} \widetilde{C}^{*}\right)^{1 / 2} \\
\left(\widetilde{C} \widetilde{C}^{*}\right)^{1 / 2}(\varphi)
\end{array}\right) \\
& \quad=\left(\begin{array}{c}
\widetilde{B}(\varphi) \Phi^{-1}(\varphi) \\
\widetilde{C}(\varphi) \Phi^{-1}(\varphi)
\end{array}\right)=\left(\begin{array}{c}
\widetilde{B}^{\prime} \\
\widetilde{C}^{\prime}
\end{array}\right)(\varphi) .
\end{aligned}
$$

Таким образом, если существует комплексный росток $r^{n}(\varphi), \varphi \in \Lambda^{k}$, то всегда существует базис на плоскости $r^{n}(\varphi)$, составленный из векторов матрищы

$$
\left(\begin{array}{l}
B \\
C
\end{array}\right)
$$

(8.1), удовлетворяющий условиям (8.2), который является гладким базисом, заданным на торе $\Lambda^{k}$ : он определяется в силу (8.1) матрицей

$$
\left(\begin{array}{c}
\widetilde{B} \\
\widetilde{C}
\end{array}\right)
$$

(штрихи у матриц $\widetilde{B}^{\prime}$ и $\widetilde{C}^{\prime}$ опускаем). Обозначим через $V(\varphi)((2 n) \times(n-k))$-матрицу, заданную на торе соотношением

$$
V(\varphi)=R(\varphi)\left(\begin{array}{c}
\widetilde{B} \\
\widetilde{C}
\end{array}\right)(\varphi)
$$

Возьмем комплексносопряженную матрищу

$$
\bar{V}(\varphi)=R(\varphi)\left(\begin{array}{c}
\overline{\tilde{B}} \\
\tilde{\tilde{C}}
\end{array}\right)(\varphi)
$$


тогда из (8.4) следует, что эти матрицы нормированы условием

$$
V^{*}(\varphi) J V(\varphi)=2 i E_{n-k}
$$

и росток $r^{n}(\varphi), \varphi \in \Lambda^{k}$, задается матрицами

$$
\left(\begin{array}{l}
B \\
C
\end{array}\right)(\varphi)=\left(V(\varphi), Y_{\varphi}(\varphi)\right)=\left(R(\varphi)\left(\begin{array}{l}
\widetilde{B} \\
\widetilde{C}
\end{array}\right)(\varphi), Y_{\varphi}(\varphi)\right)=\left(R(\varphi) \mathscr{M}(\varphi), Y_{\varphi}(\varphi)\right) .
$$

Воспользуемся инвариантностью комплексного ростка. Из условия $4^{\prime}$ (инвариантности фактора комплексного ростка $\tilde{r}^{n-k}$ ) следует, что для базиса $V(\varphi)$ на $\tilde{r}^{n-k}(\varphi), \varphi \in \Lambda^{k}$, справедливо равенство $d g_{H}^{t} V(\varphi)=V(\varphi+\omega t) U(t, \varphi)$, где $U(t, \varphi)$ - невырожденная $((n-k) \times(n-k))$-матрица $U(0, \varphi)=E_{n-k}$, являющаяся унитарной в силу того, что условие (8.5) сохраняется при сдвиге $d g_{H}^{t}$ вдоль траекторий системы (2.2). Обозначим $Y(t, \varphi)=d g_{H}^{t} Y(\varphi)$, где матрица $Y(\varphi)$ вместе с матрицами $R(\varphi), Y_{\varphi}(\varphi)$ образуют симплектический базис в $\mathbb{C}_{\varphi} \mathbb{R}_{p, x}^{2 n}$, введенньй вьше. Тогда вектор-столбцы $((2 n) \times(2 n))$-матрицы

$$
\Phi(t, \varphi)=\left(V(\varphi+\omega t) U(t, \varphi), \bar{V}(\varphi+\omega t) \bar{U}(t, \varphi), Y_{\varphi}(\varphi+\omega t), N(t, \varphi)\right)
$$

образуют базис в пространстве решений системы в вариациях (2.2) в комплексификации касательного пространства $T \mathbb{R}_{p, x}^{2 n}$. Тем самьм, представление матрицы Коши системы (2.2) в виде (2.5) доказано. При этом условия (2.6) суть следствия условий (8.5) и условий нормировки 1)-3), поскольку они сохраняются в силу гамильтоновой системы (2.2).

Доказательство второго утверждения леммы (о существовании комплексного ростка на $\Lambda^{k}$ ) непосредственно следует из формул $(2.5),(2.6)$ и заключается в проверке аксиом комплексного ростка $(2.1)-(2.3)$ на плоскости $r^{n}(\varphi) \in \mathbb{C}_{w, z}^{2 n}$, заданной в каждой точке $\varphi \in \Lambda^{k}$ формулой $(3.6)$.

ДокАЗАТЕЛЬСТво тЕоРЕмы 1. Первое утверждение теоремы устанавливается прямым вычислением матрицы Якоби $\Gamma\left(y^{\prime}\right)=\partial y / \partial y^{\prime}$ в силу явных формул замены переменных $(3.1),(3.2)$ и проверкой свойства симплектичности матрицы $\Gamma\left(y^{\prime}\right):{ }^{t} \Gamma\left(y^{\prime}\right) J \Gamma\left(y^{\prime}\right)=J$.

Для доказательства второго утверждения подставим (3.1) в гамильтониан $H(y)$ и разложим его в ряд Тейлора по переменным $\xi$ и $\bar{\xi}$ с точностью до $O\left(\|\xi\|^{3}\right),\|\xi\| \rightarrow 0$. Имеем

$$
\begin{aligned}
H(y)= & H(Y(\varphi, I))+\left\langle H_{y}(Y(\varphi, I)), \frac{V \xi+\bar{V} \bar{\xi}}{2}\right\rangle+\left\langle H_{y}(Y(\varphi, I)), Y_{I}(\varphi, I) g\right\rangle \\
& +\frac{1}{8}\left\langle V \xi+\bar{V} \bar{\xi}, H_{y y}(Y(\varphi, I))(V \xi+\bar{V} \bar{\xi})\right\rangle+O\left(\|\xi\|^{3}\right) .
\end{aligned}
$$

Слагаемые, линейные по $\xi$ и $\bar{\xi}$, обращаются в нуль в силу свойств $(2.6)$ для матрищ $V=V(\varphi, I)$ и $Y_{\varphi}=Y_{\varphi}(\varphi, I)$ и инвариантности тора $\Lambda^{k}(I)$. Действительно, в силу гамильтоновой системы $(2.1)$, которая в координатах $y={ }^{t}(p, x)$ имеет вид $\dot{y}=J H_{y}(y)$, для траектории $\gamma_{\varphi}(t)=\left\{y=Y(\omega t+\varphi, I), \varphi \in \Lambda^{k}, t \in \mathbb{R}\right\} \in \Lambda^{k}$ при $t=0$ получим

$$
J H_{y}(Y(\varphi, I))=\left.\frac{d}{d t} Y(\omega t+\varphi, I)\right|_{t=0}=\dot{Y}(\varphi, I)=\left\langle\omega(I), \frac{\partial}{\partial \varphi}\right\rangle Y(\varphi, I) .
$$


Отсюда и из (2.6) следует, что

$$
\begin{aligned}
\left\langle H_{y}(Y(\varphi, I)), V \xi\right\rangle & =\left\langle V H_{y}(Y(\varphi, I)), \xi\right\rangle=-\left\langle{ }^{t} V J\left\langle\omega(I), \frac{\partial}{\partial \varphi}\right\rangle Y(\varphi, I), \xi\right\rangle \\
& =-\sum_{l=1}^{k} \omega_{l}(I)\left\langle{ }^{t} V J Y_{\varphi_{l}}(\varphi, I), \xi\right\rangle=0 .
\end{aligned}
$$

Преобразуем третье слагаемое в разложении (8.6), воспользовавшись тем, что в координатах действие-угол $(I, \varphi)$ частоты условно-периодического движения на торе $\Lambda^{k}(I)$ имеют вид

$$
\omega_{l}(I)=\left.\frac{\partial}{\partial I_{l}} H\right|_{\Lambda^{k}(I)}=\frac{\partial}{\partial I_{l}} H(Y(\varphi, I))=\left\langle H_{y}(Y(\varphi, I)), Y_{I_{l}}(\varphi, I)\right\rangle, \quad l=1,2, \ldots, k .
$$

Отсюда и из формулы (8.7) следуют равенства $\left\langle J Y_{\varphi_{l}}(\varphi, I), Y_{I_{j}}(\varphi, I) g\right\rangle=-\delta_{l j}$. Следовательно,

$$
\begin{aligned}
\left\langle H_{y}\left(Y_{I}(\varphi, I)\right), Y_{I}(\varphi, I) g\right\rangle & =\sum_{l=1}^{k} \sum_{j=1}^{k}\left\langle J Y_{\varphi_{l}}, Y_{I_{j}} g_{j}\right\rangle \\
& =-\sum_{l=1}^{k} \sum_{j=1}^{k} \omega_{l}(I) \delta_{l j} g_{j}=-\langle\omega(I), g(\varphi, I, \xi, \bar{\xi})\rangle .
\end{aligned}
$$

Обозначим, как и в $(8.7)$, через $\dot{V}(\varphi, I)$ производную матрицы $V(\varphi, I)$ в точке $\varphi \in \Lambda^{k}$ вдоль гамильтонова векторного поля $J H_{y}$, суженного на тор $\Lambda^{k}$, т.е. $\dot{V}(\varphi, I)=\langle\omega(I)$, $\partial / \partial \varphi\rangle V(\varphi, I)$. Тогда из $(8.8)$ и определения вектора $g(\varphi, I, \xi, \bar{\xi})(3.2)$ для третьего слагаемого в (8.6) получим

$$
\left\langle H_{y}(Y(\varphi, I)), Y_{I}(\varphi, I) g\right\rangle=\frac{1}{8}\left\langle\xi,{ }^{t} V J \dot{V} \xi\right\rangle+\frac{1}{8}\left\langle\bar{\xi},{ }^{t} \bar{V} J \dot{\bar{V}} \bar{\xi}\right\rangle+\frac{1}{8}\left\langle\xi,{ }^{t} V J \dot{\bar{V}} \bar{\xi}\right\rangle+\frac{1}{8}\left\langle\bar{\xi},{ }^{t} \bar{V} J \dot{V} \xi\right\rangle .
$$

Воспользуемся теперь леммой 1 , в силу которой вектор $\eta(\varphi, t, \xi)=V(\omega t+\varphi, I) U(\varphi, t) \xi$ так же, как и вектор $\bar{\eta}(\varphi, t, \xi)$, является решением уравнения в вариациях $(2.2): \dot{\eta}=$ $J H_{y y}(Y(\omega t+\varphi)) \eta, \eta \in \mathbb{C}^{2 n}$. Отсюда следует, что

$$
\dot{V} \xi+\left.V \frac{d}{d t}(U \xi)\right|_{t=0}=J H_{y y}(Y(\varphi, I)) V \xi
$$

$\mathrm{У}_{\text {множив слева это равенство на матрицу }}{ }^{t} V J$ и воспользовавшись соотношением (2.6), найдем, что

$$
{ }^{t} V J \dot{V} \xi=-{ }^{t} V H_{y y}(Y(\varphi, I)) V \xi, \quad{ }^{t} \bar{V} J \dot{\bar{V}} \bar{\xi}=-{ }^{t} \bar{V} H_{y y}(Y(\varphi, I)) \bar{V} \bar{\xi}
$$

Заметим теперь, что последние два члена в правой части формулы (8.9) равны друг другу. Действительно, в силу третьего свойства матриц $V=V(\varphi, I)$ из (2.6) ${ }^{t} \bar{V} J V=$ $2 i E_{n-k}$, которое сохраняется при дифференцировании вдоль траектории системы $(2.1)$ на торе $\Lambda^{k}$, имеем равенство ${ }^{t} \dot{\bar{V}} J V=-{ }^{t} \bar{V} J \dot{V}$. Отсюда с учетом того, что ${ }^{t} J=-J$, 
получаем требуемое равенство. Таким образом, третий член в разложении (8.6) преобразуется с учетом равенств (8.10) к следующему виду:

$$
\begin{aligned}
\left\langle H_{y}(Y(\varphi, I)), Y_{I}(\varphi, I) g\right\rangle= & -\frac{1}{8}\left\langle\xi,{ }^{t} V H_{y y}(Y(\varphi, I)) V \xi\right\rangle-\frac{1}{8}\left\langle\bar{\xi},{ }^{t} \bar{V} H_{y y}(Y(\varphi, I)) \bar{V} \bar{\xi}\right\rangle \\
& +\frac{1}{4}\left\langle\bar{\xi},{ }^{t} \bar{V} J \dot{V} \xi\right\rangle .
\end{aligned}
$$

Подстановка этого выражения в разложение (8.6) приводит гамильтониан $H(y)$ к нормальной форме (3.4) с матрицей $A(\varphi, I)(3.5)$.

ДОКАЗАТЕЛЬСТВО СЛЕДСТВИЯ ИЗ ТЕОРЕМЫ 1 (п. 4). В силу определения матрищ $Y_{\varphi}(\varphi, I)$ и $V(\varphi, I)$, образуюших базис в слое нормального расслоения над точкой $\varphi$, $\varphi \in \Lambda^{k}(I)$, многообразие $\Lambda^{n}(I, \rho)$ при $|\rho| \neq 0$ является гладким $n$-мерньм многообразием $\left(\operatorname{rank} \partial^{2} Y / \partial \varphi \partial \eta=n\right)$. Очевидно, что в силу определения $(4.1) \Lambda^{n}(I, \rho)-n$-мерньй тор (при $|\rho| \neq 0$ ) с угловыми координатами $\varphi_{j}, \eta_{l}, j=1,2, \ldots, k, l=1,2, \ldots, n-k$. Этот тор лагранжев, поскольку он является вложением в $\mathbb{R}_{y}^{2 n} n$-мерного лагранжева тора $\widetilde{\Lambda}^{n}=\left(\mathbb{R}_{\varphi}^{k} \times \mathbb{R}_{\eta}^{n-k} / \mathbb{Z}^{n}\right) \times(I=$ const, $\rho=$ const $)$ с помощью отображения $F: \widetilde{\Lambda}^{n} \rightarrow \Lambda^{n}(I, \rho)$, где $F$ - каноническое (в силу леммы 1 ) отображение, определенное формулой (3.1). Далее, введем функцию

$$
\begin{aligned}
Y_{\rho} \equiv & Y_{\rho}(\omega(I) t+\varphi, \beta(I) t+\eta, I, \rho) \\
\equiv & Y(\omega(I) t+\varphi, I)+\operatorname{Re}\left(V(\omega(I) t+\varphi, I) U_{0}(\omega(I) t+\varphi, I) e^{i(\hat{\beta} t+\hat{\eta})} \sqrt{\frac{\rho}{2}}\right) \\
& +X_{I}(\omega(I) t+\varphi, I) f\left(\omega(I) t+\varphi, e^{i(\hat{\beta} t+\hat{\eta})} \sqrt{\frac{\rho}{2}}\right),
\end{aligned}
$$

где $\omega(I)-k$-мерный вектор частот условно-периодического движения на изотропном инвариантном торе $\Lambda^{k}(I), \beta(I)-(n-k)$-мерньй вектор чисел вращения системы в вариациях $(3.6)$ на торе $\Lambda^{k}(I)$ и $\eta=\left(\eta_{1}, \eta_{2}, \ldots, \eta_{n-k}\right)$ - угловые “нормальные" по отношению к $\Lambda^{k}(I)$ переменные.

Функция $Y_{\rho}$ удовлетворяет системе Гамильтона $\dot{y}=J H_{y}(y)$ по $\bmod O(|\rho|)$. Это немедленно следует из результатов леммы 2 (см. ниже). В симплектических координатах $y^{\prime}=(\varphi, I, \xi, \bar{\xi})$ исходная система Гамильтона с гамильтонианом $H(y)$ (с нормальной формой (3.4)) имеет вид

$$
\dot{\varphi}=\omega(I)+O\left(|\xi|^{2}\right), \quad \dot{I}=O\left(|\xi|^{2}\right), \quad \dot{\xi}=2 i \frac{\partial H}{\partial \bar{\xi}}=i A(\omega(I) t+\varphi, I)+O\left(|\xi|^{2}\right)
$$

при $|\xi| \rightarrow 0$. В этих координатах для любой точки $(\varphi, \tilde{\xi})$ на торе $\Lambda^{n}(\rho, I)$, где $\tilde{\xi}=e^{i \eta} \sqrt{\rho}$, функции $\varphi_{t}(\varphi)=\omega(I) t+\varphi, \varphi \in \Lambda^{k}, \xi(t, \varphi)=U_{0}(\omega(I) t+\varphi, I) e^{i \hat{\beta} t} \tilde{\xi}$ удовлетворяют в силу условий теоремы этой системе по $\bmod O(|\rho|)$, где $|\rho|=|\xi|^{2}$. Значение гамильтониана $H(y)$ на лагранжевом торе $\Lambda^{n}(I, \rho)(4.1)$ вычисляется элементарно, исходя из представления (3.4) и свойства приводимости.

Доказательство теоремы 2 опирается на следующее утверждение. Обозначим через $R(\varphi, \eta)(n \times n)$-матрицу на торе $\Lambda^{n}(I, \rho)$ вида

$$
R(\varphi, \eta)=\left(X_{\varphi}(\varphi),-\operatorname{Im}\left(\widetilde{C}(\varphi) e^{i \hat{\eta}}\right)\right)-i \varepsilon\left(P_{\varphi}(\varphi),-\operatorname{Im}\left(\widetilde{B}(\varphi) e^{i \hat{\eta}}\right)\right)
$$


Лемма 2. Матрица $R(\varphi, \eta)$ при $\varepsilon>0$ невырождена, и приращения аргументов определителя этой матрицы и якобиана $J(\varphi, \eta)(5.5)$ вдоль замкнутого пути на $\Lambda^{n}(I, \rho)$ равны.

ДокАЗАТЕЛЬСтво. Предположим, что $\operatorname{det} R(\varphi, \eta)=0$ для некоторого $\varepsilon>0$. Тогда существует вектор $f$ такой, что $R(\varphi, \eta) f=0$. Обозначим

$$
C_{1}=C_{1}(\varphi, \eta)=\left(X_{\varphi}(\varphi),-\operatorname{Im}\left(\widetilde{C}(\varphi) e^{i \hat{\eta}}\right)\right), \quad B_{1}=B_{1}(\varphi, \eta)=\left(P_{\varphi}(\varphi),-\operatorname{Im}\left(\widetilde{B}(\varphi) e^{i \hat{\eta}}\right)\right) .
$$

Тогда имеем $C_{1} f=i \varepsilon B_{1} f$. Из условия лагранжевости тора $\Lambda^{n}(I, \rho)$ следует, что ${ }^{t} B_{1} C_{1}$ $={ }^{t} C_{1} B_{1}$. Отсюда имеем равенство $\left\langle B_{1} \bar{f}, B_{1} f\right\rangle=\left\langle C_{1} \bar{f}, B_{1} f\right\rangle$ или с учетом выбора вектора $f: i\left\langle B_{1} \bar{f}, B_{1} f\right\rangle=-i\left\langle B_{1} \bar{f}, B_{1} f\right\rangle$. Следовательно, $\left\langle B_{1} \bar{f}, B_{1} f\right\rangle=0$ и $B_{1} f=0$. Но тогда и $C_{1} f=0$. Тем самым

$$
\operatorname{rank}\left(\begin{array}{c}
B_{1} \\
C_{1}
\end{array}\right)<n
$$

что противоречит тому, что $\Lambda^{n}(I, \rho) n$-мерньй лагранжев тор.

Для доказательства второго утверждения леммы выразим якобиан $J(\varphi, \eta)$ через $\operatorname{det} R(\varphi, \eta)$. Из формул (5.3) найдем при $\rho \rightarrow 0$ следующие асимптотики:

$$
\begin{aligned}
& \frac{\partial \tilde{X}}{\partial(\varphi, \eta)}=\left(X_{\varphi}(\varphi)+\operatorname{Re}\left(\widetilde{C}_{\varphi}(\varphi) e^{i \hat{\eta}} \sqrt{\rho}\right), \operatorname{Re}\left(\widetilde{C}(\varphi) i e^{i \hat{\eta}} \sqrt{\rho}\right)\right)+O\left(|\rho|^{2}\right), \\
& \frac{\partial \tilde{P}}{\partial(\varphi, \eta)}=\left(P_{\varphi}(\varphi)+\operatorname{Re}\left(\widetilde{B}_{\varphi}(\varphi) e^{i \hat{\eta}} \sqrt{\rho}\right), \operatorname{Re}\left(\widetilde{C}(\varphi) i e^{i \hat{\eta}} \sqrt{\rho}\right)\right)+O\left(|\rho|^{2}\right),
\end{aligned}
$$

где $\sqrt{\rho}=\operatorname{diag}\left(\sqrt{\rho_{l}}\right), l=1,2, \ldots, n-k$, и, следовательно, матрица $D(\varphi, \eta)(5.4)$ представима в виде

$$
D(\varphi, \eta)=R(\varphi, \eta) \sqrt{\rho}+\left(0, \operatorname{Re}\left(\widetilde{C}_{\varphi}(\varphi)-i \varepsilon \widetilde{B}_{\varphi}(\varphi)\right) e^{i \hat{\eta}} \sqrt{\rho}\right)+O\left(|\rho|^{2}\right) .
$$

Отсюда при $|\rho| \rightarrow 0$ следует, что

$$
J(\varphi, \eta)=\left(\prod_{l=1}^{n-k} \sqrt{\rho_{l}}\right) \operatorname{det} R(\varphi, \eta)(1+O(\sqrt{\rho})),
$$

где оценка равномерна по $(\varphi, \eta) \in[0,2 \pi]^{n}$. Поскольку прирашение $\operatorname{Arg} J(\varphi, \eta)$ есть непрерьвная функция $\rho$, то полученное представление для якобиана $J(\varphi, \eta)$ доказывает требуемое утверждение леммы.

Определим теперь семейство $(n \times(n-k))$-матриц $\widetilde{C}_{\delta}(\varphi, \eta)$ и $\widetilde{B}_{\delta}(\varphi, \eta)$, зависящих от параметра $\delta \in[0,1]$, следующими формулами:

$$
\widetilde{C}_{\delta}(\varphi, \eta)=\frac{\widetilde{C}(\varphi) e^{i \hat{\eta}}-\delta \overline{\widetilde{C}}(\varphi) e^{-i \hat{\eta}}}{2 i}, \quad \widetilde{B}_{\delta}(\varphi, \eta)=\frac{\widetilde{B}(\varphi) e^{i \hat{\eta}}-\delta \overline{\widetilde{B}}(\varphi) e^{-i \hat{\eta}}}{2 i}
$$

Рассмотрим два семейства $(n \times n)$-матрищ

$$
C_{\delta}(\varphi, \eta)=\left(X_{\varphi}(\varphi), \widetilde{C}_{\delta}(\varphi, \eta)\right), \quad B_{\delta}(\varphi, \eta)=\left(P_{\varphi}(\varphi), \widetilde{B}_{\delta}(\varphi, \eta)\right)
$$

где функции $X(\varphi), P(\varphi)$ задают изотропный тор $\Lambda^{k}(I)$. 
Лемма 3. Пусть $\eta \in[0,2 \pi]^{n-k}$ фиксировано $и \delta \neq 1$. Тогда матрииы $C_{\delta}(\varphi, \eta)$ $u B_{\delta}(\varphi, \eta)$ определяют базис на комплексном ростке $r_{\delta}^{n}(\varphi), \varphi \in \Lambda^{k}(I)$, причем матрии, а

$$
D_{\delta}(\varphi, \eta)=C_{\delta}(\varphi, \eta)-i \varepsilon B_{\delta}(\varphi, \eta)
$$

невырохсена при $\varepsilon>0$.

ДокАЗАТЕЛЬСТво. Условия лагранжевости (8.2a) и диссипативности (8.2b) для матрищ $C_{\delta}(\varphi, \eta), B_{\delta}(\varphi, \eta)$ проверяются непосредственньм вычислением с учетом свойств лагранжевости и диссипативности для матриц $\widetilde{C}(\varphi), \widetilde{B}(\varphi)$ - базиса на факторе комплексного ростка $\tilde{r}^{n-k}(\varphi), \varphi \in \Lambda^{k}(I)$; при этом условие диссипативности имеет вид

$$
\begin{gathered}
\frac{1}{2 i}\left(\widetilde{C}_{\delta}^{*}(\varphi, \eta) \widetilde{B}_{\delta}(\varphi, \eta)-\widetilde{B}_{\delta}^{*}(\varphi, \eta) \widetilde{C}_{\delta}(\varphi, \eta)\right)=\frac{1}{2 i}\left(\widetilde{C}^{*}(\varphi) \widetilde{B}(\varphi)-\widetilde{B}^{*}(\varphi) \widetilde{C}(\varphi)\right)\left(1-\delta^{2}\right)>0, \\
\delta \neq 1 .
\end{gathered}
$$

Докажем невырожденность матрицы $D_{\delta}(\varphi, \eta)$. Предположим, что $\operatorname{det} D_{\delta}(\varphi, \eta)=0$ при некотором $\varepsilon>0$. Тогда существует вектор $u \in \mathbb{C}^{n}$ такой, что $C_{\delta}(\varphi, \eta) u=i \varepsilon B_{\delta}(\varphi, \eta) u$. Отсюда имеем равенство

$$
\frac{1}{2 i}\left\langle\bar{u},\left(C_{\delta}^{*} B_{\delta}-B_{\delta}^{*} C_{\delta}\right) u\right\rangle=-\varepsilon\left\langle\bar{B}_{\delta} \bar{u}, B_{\delta} u\right\rangle .
$$

В силу неотрицательности левой части равенства следует, что $B_{\delta} u=0$. Но тогда и $C_{\delta} u=0$. Это означает, что матрица

$$
\left(\begin{array}{c}
P_{\varphi}(\varphi) \\
X_{\varphi}(\varphi)
\end{array}\right)
$$

имеет ранг меньше $k$. Это противоречит условию о том, что $\Lambda^{k}(I)-($ гладкий) $k$-мерный тор.

ДОКАЗАТЕЛЬСТВО ТЕОРЕМЫ 2 следует непосредственно из результатов лемм 2,3 и того факта, что в силу определения матрищ $D_{\delta}(\varphi, \eta)(8.13), D(\varphi, \eta)(5.8),(5.9)$ и матрицы $R(\varphi, \eta)(8.12)$ справедливы равенства

$$
\left.D_{\delta}\right|_{\delta=0}(\varphi, \eta)=\frac{1}{2 i} D(\varphi, \eta),\left.\quad D_{\delta}\right|_{\delta=1}(\varphi, \eta)=-R(\varphi, \eta) .
$$

Действительно, для комплексной функции $F(\varphi, \eta, \varepsilon, \delta)=\operatorname{det} D_{\delta}(\varphi, \eta)$ при $\varepsilon>0$ и не равном нулю $\delta \in[0,1)$ приращение ее аргумента $\operatorname{Arg} F$ по циклу $\Gamma_{j}(5.8 \mathrm{a})$ (кратное $2 \pi$ ) является непрерывной функцией $\delta$, не зависящей от $\varepsilon>0$. Следовательно, в силу равенств (8.14) для $\varphi$-циклов $\Gamma_{j}$ имеем

$$
\Delta_{\Gamma_{j}} \operatorname{Arg} \operatorname{det} D_{\delta}(\varphi, \eta)=\Delta_{\Gamma_{j}} \operatorname{Arg} \operatorname{det} D(\varphi, \eta)=\Delta_{\Gamma_{j}} \operatorname{Arg} \operatorname{det} R(\varphi, \eta) .
$$

Для $\eta$-циклов $\gamma_{l}$ отсюда и из формулы (5.10) найдем, что индекс Маслова цикла $\gamma_{l}$ выражается формулой

$$
m_{l}=\frac{1}{\pi} \Delta_{\gamma_{l}} \operatorname{Arg} J(\varphi, \eta)=\frac{1}{\pi} \Delta_{\gamma_{l}} \operatorname{Arg} \operatorname{det} R(\varphi, \eta)=\frac{1}{\pi} \operatorname{Arg} J(\varphi, \eta)=2
$$

для любого $l=1,2, \ldots, n-k$.

Доказательство теоремы 3 мы здесь не приводим, так как это потребовало бы новых вспомогательных конструкций; в частности, (локального) канонического оператора Маслова на изотропном торе с комплексным ростком, формул коммутации псевдодифференциального оператора $\widehat{H}$ с “экспонентой” и метода стационарной фазы [2], [15], [16], [51]. 
ДОКАЗАТЕЛЬСТВО ТЕОРЕМЫ 5. Воспользуемся тем, что однородные полиномы

$$
\left\{\frac{\xi^{\alpha}}{\sqrt{\alpha !}}, \alpha \in\left(\mathbb{Z}^{+}\right)^{n-k}, \xi \in \mathbb{C}^{n-k}\right\}
$$

где $\alpha=\left(\alpha_{1}, \ldots, \alpha_{n-k}\right)$ - мультииндекс, образуют ортонормированньй базис в фоковском пространстве $F_{2}$, т.е. $\left\langle\alpha \mid \alpha^{\prime}\right\rangle=\delta_{\alpha \alpha^{\prime}}$, и будем искать решение задачи (6.4) в виде ряда

$$
f_{\lambda}(\varphi, \bar{\xi})=\sum_{\alpha} C_{\alpha}^{\lambda}(\varphi) \frac{\bar{\xi}^{\alpha}}{\sqrt{\alpha !}}, \quad \alpha \in\left(\mathbb{Z}^{+}\right)^{n-k}, \quad \xi \in \mathbb{C}^{n-k}
$$

Подставим функцию $f_{\lambda}(\varphi, \bar{\xi})$ в уравнение $(6.4)$ и воспользуемся системой $(6.2)$, положив $t=0$. Тогда получим

$$
\begin{aligned}
& \sum_{\alpha} i \dot{C}_{\alpha}^{\lambda}(\varphi) \bar{\xi}^{\alpha}+\sum_{j=1}^{n-k} \sum_{m=1}^{n-k} \sum_{\alpha} C_{\alpha}^{\lambda}(\varphi) \alpha_{m} A_{j m} \bar{\xi}_{1}^{\alpha_{1}}, \ldots, \bar{\xi}_{j}^{\alpha_{j}-1}, \ldots, \bar{\xi}_{m}^{\alpha_{m}+1}, \ldots, \bar{\xi}_{\alpha_{m-k}}^{\alpha_{n-k}} \\
& \quad=\lambda \sum_{\alpha} C_{\alpha}^{\lambda}(\varphi) \bar{\xi}^{\alpha}
\end{aligned}
$$

Воспользовавшись теперь ортогональностью функций $\bar{\xi}^{\alpha} / \sqrt{\alpha}$, после несложных преобразований мультииндексов получим спектральную задачу (6.13).

9. Заключительные замечания. Проведенные в этой статье исследования порождают некоторые новые интересные, на наш взгляд, вопросы. К их числу относятся, в частности, вопросы о спектрах динамического потока и соответствующей системы уравнений с почти периодическими коэффициентами “в нерегулярном случае". Такие вопросы возникают, как уже отмечалось, в задачах со "слабым хаосом", и, вероятно, на такие задачи целесообразно посмотреть с точек зрения подходов, предложенных в [67]-[72]. Другая, противоположная ситуация возникает в "резонансньх случаях", когда частоты движения на торе соизмеримы и асимптотические собственные значения оказьваются вырожденными с точностью до $O\left(h^{2}\right)$. В некоторых физически интересных задачах такое "асимптотическое вырождение", по всей видимости, снимается при построении высших приближений асимптотики ${ }^{13}$. При этом соответсвующие поправки возникают как из классической, так и квантовой механики. Ясно, что нахождение "классических" поправок связано с построением нормальных форм высокого порядка, а нахождение "квантовых" поправок лежит в круге задач некоммутативного анализа и “деформационного" квантования. Здесь могут оказаться полезными подходы, изложенные в [4], [5], [34]-[47], [73], [74]. Наконец, было бы интересно взглянуть с точки зрения теории нормальных форм на построенные в [75] квазимоды спектральных задач для уравнений с малой диффузией.

Благодарности. Авторы благодарят за полезные обсуждения А.Д. Брюно, Ю.М. Воробьева, В.В. Козлова, В.П. Маслова, А.И. Нейштадта, В.И. Оселедца, Я. Г. Синая и А.И. Шафаревича.

\footnotetext{
${ }^{13}$ Подчеркнем, что речь здесь идет о степенном по параметру $h$ вырождении, а не об экспоненциально малом расщеплении собственных значений, обусловленных туннельными эффектами.
} 


\section{СПИСОК ЦИТИРОВАННОЙ ЛИТЕРАТУРЫ}

[1] Маслов В.П. Теория возмущений и асимптотические методы. М.: Изд-во МГУ, 1965; Асимптотические методы и теория возмущений. М.: Наука, 1988.

[2] Маслов В. П. Операторные методы. М.: Наука, 1973.

[3] Маслов В. П., Федорюк М. В. Квазиклассическое приближение для уравнений квантовой механики. М.: Наука, 1976.

[4] Карасев М. В., Маслов В. П. Асимптотическое и геометрическое квантование // УМН. 1984. Т. 39. № 6 . C. $115-173$.

[5] Карасев М. В., Маслов В. П. Нелинейные скобки Пуассона. М.: Наука, 1991.

[6] Арнольд В.И. Моды и квазимоды // Функцион. анализ и его прилож. 1972. Т. 6. № 2. C. $12-20$.

[7] Lazutkin V.F. KAM Theory and Semiclassical Approximations to Eigenfunctions. Ergeb. Math. Grenzgeb. (3). V. 24. Berlin: Springer-Verlag, 1993.

[8] Крахнов А. Д. Построение асимптотики собственных чисел оператора Лапласа, отвечающих невырожденному инвариантному тору геодезического потока // Методы качественной теории дифференциальных уравнений. Вып. 1. Горький: Изд-во ГГУ, 1969. С. 66-74.

[9] Colin de Verdière Y. Spectre conjoint d'operateurs pseudo-differentiels qui commitent II. Le cas integrable // Math. Z. 1980. V. 171. №1. P. 51-73.

[10] Косыгин Д. М., Минасов А. А., Синай Я. Г. Статистические свойства спектра операторов Бельтрами-Лапласа на поверхностях Лиувилля // УМН. 1993. Т. 48. № 5. С. 3-130.

[11] Болсинов А. В., Фоменко А. Т. Введение в топологию интегрируемых систем. М.: Наука, 1997.

[12] Фоменко А. Т. Симплектическая геометрия. Методы и приложения. М.: МГУ, 1988.

[13] Маслов В. П. Комплексный метод ВКБ в нелинейных уравнениях. М.: Наука, 1977.

[14] Маслов В. П., Шведов О. Ю. Метод комплексного ростка в квантовой теории поля. М.: УРСС, 1998.

[15] Белов В. В., Доброхотов С. Ю. Канонический оператор Маслова на изотропных многообразиях с комплексным ростком и его приложение к спектральным задачам // Докл. АН CCCP. 1988. T. 298. № 5. C. 1037-1042.

[16] Белов В. В., Доброхотов С. Ю. Квазиклассические асимптотики Маслова с комплексными фазами. I // ТМФ. 1992. Т. 92. № 2. С. 215-254.

[17] Бабич В. М. Собственные функции, сосредоточенные в окрестности замкнутой геодезической. Записки научных семинаров ЛОМИ. Т. 9. Ленинград, 1968.

[18] Бабич В. М., Булдырев В. С. Асимптотические методы в задачах дифракции коротких волн. М.: Наука, 1972.

[19] Доброхотов С. Ю., Маслов В. П. Некоторые приложения теории комплексного ростка к уравнениям с мальм параметром // Совр. пробл. математики. Т. 5. М.: ВИНИТИ, 1975. C. $141-207$.

[20] Доброхотов С. Ю., Маслов В.П. Многомерные ряды Дирихле в многомерной задаче об асимптотике спектральных серий нелинейных эллиптических операторов // Совр. пробл. математики. Т. 23. М.: ВИНИТИ, 1983. С. 137-220.

[21] Ralston J. V. On the construction of quasimodes associated with stable periodic orbits // Comm. Math. Phys. 1976. V. 51. P. 219-242.

[22] Valiño B., Доброхотов С. Ю., Нехорошев Н. Н. Комплексный росток в системах с одной циклической переменной // УМН. 1984. Т. 39. № 3. С. 233-239.

[23] Доброхотов С. Ю., Мартинес-Оливе В. Замкнутые траектории и двумерные торы в квантовой спектральной задаче для трехмерного ангармонического осциллятора // Тр. ММО. 1997. T. 58. C. 3-87.

[24] Dobrokhotov S. Yu., Olivé V. M., Shafarevich A. I. Closed trajectories and twodimensional tori in the quantum problem for a three-dimensional resonant anharmonic oscillator // Russian J. Math. Phys. 1995. V. 3. № 1. P. 133-138.

[25] Доброхотов С. Ю., Шафаревич А. И. Квазиклассическое квантование изотропных многообразий гамиль тоновых систем // Топологические методы в теории гамильтоновых систем. М.: Факториал, 1998. 
[26] Dobrokhotov S. Yu., Shafarevich A. I. Quantum selection of isotropic tori of partially integrable hamiltonian systems in quasiclassical approximation // Russian J. Math. Phys. 1998. V. 5. № 2. P. 267-272.

[27] Воробьев Ю. М. Комплексный росток Маслова, порожденный линейной связностью // Матем. заметки. 1990. Т. 48. №6. С. 29-37.

[28] Карасев М. В. Новые глобальные асимптотики и аномалии в задачах квантования адиабатических инвариантов // Функцион. анализ и его прилож. 1990. Т. 24. № 2. С. 24-36.

[29] Карасев М. В. Квантовая редукция на орбиты алгебр симметрий и задача Эренфеста. Препринт ИТФ-87-157Р. Киев: Ин-т теор. физ. АН УССР, 1988.

[30] Karasev M. V., Vorob'ev Yu. M. Symplectic curvature and Arnold form over isotropic submanifolds // Math. Sci. Contemp. Math. Its Appl. (The Math. Issues). Algebra-3. V. 21, 1995.

[31] Karasev M. V., Vorob'ev Yu. M. Adapted connections, Hamiltonian dynamics, geometric phases, and quantization over isotropic submanifolds // Coherent Transform, Quantization, and Poisson Geometry. Amer. Math. Soc. Transl. V. 187. № 2. Providence, RI: Amer. Math. Soc., 1998. P. 203-326.

[32] Vorob'ev Yu. M. Ricatti equation over torus and semiclassical quantization of multiperiodic motion // Quantization and Infinite Dimentional Systems / ed. J.-P. Antoine etc.: Plenum Press, 1994. P. 205-212.

[33] Воробьев Ю. М., Ицков В.А. Квазимоды, отвечающие почти периодическому движению устойчивого типа // Матем. заметки. 1994. Т. 55. № 5. С. 36-42.

[34] Брюно А. Д. Ограниченная задача трех тел. М.: Наука, 1990.

[35] Broer H. W., Huitema G. B., Sevryuk M. B. Quasi-Periodic Motions in Families of Dynamical Systems. Lecture Note in Math. V. 1645. Berlin: Springer, 1996.

[36] Fink A. M. Almost Periodic Differential Equations. Berlin: Springer-Verlag 1974. V. 377.

[37] Арнольд В. И. О рождении условно-периодического движения из семейства периодических движений // Докл. АН СССР. 1961. Т. 138. № 1. С. 13-15.

[38] Динабург Е. Н., Синай Я. Г. Об одномерном уравнении Шредингера с квазипериодическим потенциалом // Функцион. анализ и его прилож. 1975. Т. 9. № 4. С. 8-21.

[39] Eliasson L.H. Floquet solutions for the 1-dimensional quasi-periodic Schrödinger equation // Comm. Math. Phys. 1992. V. 146. № 3. P. 447-482.

[40] Rüssmann H. Invariant Tori in the Perturbation Theory of Weakly Non-Degenerate Integrable Hamilton Systems. Preprint. V. 14. Johannes Gutenberg-Universität Mainz, 1998.

[41] Куксин С. Б. Приводимые уравнения в вариациях и возмущения инвариантных торов гамильоновых систем // Матем. заметки. 1989. Т. 45. № 5. С. 38-49.

[42] Johnson R., Mozer J. The rotation number for almost periodic potentials // Comm. Math. Phys. 1982. V. 84. P. 403-438.

[43] Johnson R. A., Sell G. R. Smoothness of spectral subbundles and reducibility of quasiperiodic linear differential systems // J. Differential Equations. 1981. №4. P. 262-288.

[44] Avron J., Simon B. Almost periodic Hill's equation and rings of Saturn // Phys. Rev. Lett. 1981. V. 46. № 17. P. 1166-1168.

[45] Jorba A., Simó C. On quasiperiodic perturbations of elliptic equilibrium points // SIAM J. Math. Anal. 1996. V. 27. P. 1704-1737.

[46] Jorba A., Villanueva J. On the normal behavior of partially elliptic lower-dimensional tori of Hamiltonian systems // Nonlinearity. 1997. V. 10. P. 783-822.

[47] Jorba A., de la Llave R., Zou M. Linstedt series for lower dimensional tori of hamiltonian systems with three or more degrees of freedom // NATO Adv. Sci. Inst. Ser. C, Math. Phys. Sci. (St. Agaro, Spain, June 1995) / ed. C. Simo. Dordrecht: Kluwer, 1990.

[48] Арнольд В.И. Математические методы классической механики. М.: Наука, 1974.

[49] Доброхотов С. Ю. Интегрирование в квадратурах $2 n$-мерных линейных гамильоновых систем с $n$ известными косоортогональными решениями // УМН. 1998. Т. 53. № 2. С. 143-144.

[50] Маслов В. П. Асимптотические методы решения псевдодифференциальных уравнений. М.: Наука, 1987. 
[51] Козлов В.В. Симметрии, топология и резонансы в гамильоновой механике. Ижевск: Изд-во УГУ, 1995.

[52] Dubnov V. L., Maslov V.P., Nazaikinskii V.E. The complex Lagrangian germ and the canonical operator // Russian J. Math. Phys. 1995. V. 3. № 2. P. 141-190.

[53] Арнольд В. И. // Функцион. анализ и его прилож. 1967. Т. 1. № 1. С. 1-114.

[54] Dobrokhotov S. Yu., Krakhnov A. D., Vorob'ev Yu. M. On topological quantization conditions for isotropic submanifolds of non-complete dimension // Proc. Int. Topological Conf. Baku, 1987. P. 75.

[55] Vorob'ev Yu. M., Nazaikinskii V. E. On the cycle of singularities of isotropic submanifolds // Russian J. Math. Phys. 1997. V. 5. № 1. P. 1-4.

[56] Нехорошев Н. Н. Теорема Пуанкаре-Ляпунова-Лиувилля-Арнольда // Функцион. анализ и его прилож. 1994. Т. 28. С. 67-69.

[57] Нехорошев Н. Н. Переменные действия-угол и их обобщение // Тр. ММО. 1972. Т. 26. C. $181-198$.

[58] Белов В. В., Максимов В. А. Квазимоды двух связанных нелинейных осцилляторов // Матем. заметки. 1998. Т. 64. № 2. С. 297-301.

[59] Belov V. V., Maksimov V. A. Stable quasimodes of the Schrödinger operator with homogeneous potential // Russian J. Math. Phys. 2000. V. 7. № 3. P. 363-370.

[60] Гельфанд И.М., Лидский В.Б. О структуре областей устойчивости линейных канонических систем дифференциальньх уравнений с периодическими коэффициентами // УМН. 1955. T. 10. № 1. C. 3-40.

[61] Березин Ф. А., Шубин М. А. Уравнение Шредингера. М.: Изд-во МГУ, 1983.

[62] Корнфельд И. П., Синай Я. Г., Фомин С. В. Эргодическая теория. М.: Наука, 1980.

[63] Voros A. The WKB-Maslov method for nonseparable systems // Ann. Inst. H. Poincaré. 1977. V. 38. № 1.

[64] Рофе-Бекетов $\Phi$. С. К вопросу об оценке роста решений канонических почти периодических систем // Матем. физика, анализ, геометрия. 1994. Т. 1. №1. С. 139-148.

[65] Рофе-Бекетов Ф. С. Константы типа Кнезера и эффективные массы для зонных потенциалов // Докл. АН СССР. 1984. Т. 276. № 2. С. 356-359.

[66] Гантмахер Ф.Р. Теория матриц. М.: Наука, 1988.

[67] Berry M. V. Semiclassical mechanics of regular and irregular motion // Chaotic Behavior of Deterministic Systems (Les Houches, Session 36, 1981): North-Holland Publishing Co., 1983. P. 171-271.

[68] Duistermaat J. J., Guillemin V.W. The spectrum of positive operators and periodic bicharacteristics // Invent. Math. 1983. V. 29. № 1. P. 63-74.

[69] Gutzwiller M. C. Chaos in Classical and Quantum Mechanics. New York: Springer-Verlag, 1992.

[70] Shnirelman A. I. On the asymptotic propertyies of eigenfunctions in the regions of chaotic motion // Addendum to book Lazutkin V.F. "KAM Theory and Semiclassical Approximations to Eigenfunctions". Ergeb. Math. Grenzgeb. (3). V. 24. Berlin: Springer-Verlag, 1993. P. 313-337.

[71] Маслов В. П., Мищенко А. С. Квазиклассическая асимптотика квазичастиц // Матем. сб. 1998. Т. 189 . № 6 . С. $85-116$.

[72] Maslov V.P. Deterministic quantum chaos for systems of bosons and fermions // Russian J. Math. Phys. 1998. V. 5. № 4. P. 473-488.

[73] Ж Куравлев В.Ф. Новый алгоритм нормализации гамильтоновых систем по Биркгофу // ПММ. 1997. Т. 61. №1. С. 12-17.

[74] Павленко Ю. Г. Гамильтоновы методы в электродинамике и квантовой механике. М.: МГУ, 1985.

[75] Доброхотов С. Ю., Колокольцов В. Н., Оливе В. М. Асимптотические устойчивые инвариантные торы векторного поля $V(x)$ и квазимоды оператора $V(x) \cdot \nabla-\varepsilon \Delta / /$ Матем. заметки. 1995. Т. 58. №6. С. 301-306. 